\title{
THE VARIETY OF MODULAR LATTICES IS NOT GENERATED BY ITS FINITE MEMBERS
}

\author{
BY
}

\author{
RALPH FREESE ${ }^{1}$
}

\begin{abstract}
This paper proves the result of the title. It shows that there is a five-variable lattice identity which holds in all finite modular lattices but not in all modular lattices. It is also shown that every free distributive lattice can be embedded into a free modular lattice. An example showing that modular lattice epimorphisms need not be onto is given.
\end{abstract}

We prove the result of the title by constructing a simple modular lattice of length six not in the variety generated by all finite modular lattices. This lattice can be generated by five elements and thus the free modular lattice on five generators, $F M(5)$, is not residually finite.

Our lattice is constructed using the technique of Hall and Dilworth [9] and is closely related to their third example. Let $F$ and $K$ be countably infinite fields of characteristics $p$ and $q$, where $p$ and $q$ are distinct primes. Let $L_{p}$ be the lattice of subspaces of a four-dimensional vector space over $F, L_{q}$ the lattice of subspaces of a four-dimensional vector space over $K$. Two-dimensional quotients (i.e. intervals) in both lattices are always isomorphic to $\boldsymbol{M}_{\boldsymbol{\omega}}$ (the two-dimensional lattice with $\omega$ atoms). Thus $L_{p}$ and $L_{q}$ may be glued together over a two-dimensional quotient via [9], and this is our lattice. Notice that if $F$ and $K$ were finite fields we could not carry out the above construction since two-dimensional quotients of $L_{p}$ would have $p^{n}+1$ atoms and those of $L_{q}$ would have $q^{m}+1$, for some $n, m \geqslant 1$. However these numbers are never equal. To some extent the proof is based on this fact.

We prove our result by letting $f$ be a homomorphism from a modular lattice $M$ onto our lattice $L$. A great deal of the structure of $L$ can be pulled back through $f$ into $M$. We then assume $M$ is residually finite and using von Neumann's theorem arrive at a contradiction similar to the one described above. K. Baker [1] and R. Wille [20] have constructed varieties of modular lattices not generated by their finite members. Using a lattice constructed by E. T. Schmidt [18] the author has shown there is a variety of modular lattices not even generated by its finite dimensional members [6]. However, Schmidt's

Received by the editors June 23, 1978 and, in revised form, September 25, 1978.

AMS (MOS) subject classifications (1970). Primary 06A30.

${ }^{1}$ This work was partially supported by the National Science Foundation Grant No. MCS77-01933. 
lattice and the one used by Baker are in the variety generated by all finite modular lattices.

The result of this paper contrasts with $R$. A. Dean's result that the variety of all lattices is generated by its finite members [3].

The first section of the paper studies (von Neumann) $n$-frames in modular lattices. The concept of an $n$-frame of characteristic $p$ is introduced. It is shown among other things that $n$-frames of characteristic $p$ are projective in the sense that they may be pulled back through homomorphisms. The results of this section will be used in a subsequent paper to show that the lattice of subgroups of the $n$-fold direct product of $\mathbf{Z}_{p}, L\left(\mathbf{Z}_{p}^{n}\right)$, is a projective modular lattice if $4 \leqslant n<\omega$ and $p$ is a prime. The second section constructs the lattice $L$ and shows that it is not in the variety generated by the finite modular lattices by the method outlined above.

Bjarni Jónsson has asked if there are any uncountable distributive sublattices of free modular lattices. (Recall that the distributive sublattices of free lattices are at most countable [7].) In the third section we show that for every infinite cardinal $\kappa$, there is a quotient sublattice (= interval) of $F M(\kappa)$ which is distributive and has cardinality $\kappa$. The lattice $L$ was originally constructed with this application in mind. In this section it is also shown that modular lattice epimorphisms, defined as in category theory, are not onto. A method for obtaining a five variable lattice identity holding in all finite modular lattices but not in all modular lattices is outlined.

In this paper we use the plus sign for the join operation and juxtaposition or dot for the meet operation, since this reduces the apparent complexity of the lattice terms. Notice that von Neumann alternated between both sets of symbols [19, p. 137]. If $a \geqslant b$ in a lattice $L$, we let $a / b=\{x \in L: b \leqslant x \leqslant$ $a$ ) and call $a / b$ a quotient or quotient sublattice of $L$ (alias interval). We say $a / b$ transposes up to $c / d$ if $a+d=c$ and $a d=b$. This is denoted $a / b>c / d$ and we also write $c / d \searrow a / b$. The quotients $a / b$ and $c / d$ are said to be projective if they are connected by a finite sequence of transposes. If $a / b>c / d$, then $a / b$ is isomorphic to $c / d$ via the map $x \mapsto x+d$. The inverse map is $y \mapsto y a$, see [2].

This paper contains some lengthy calculations. The technique usually involves first introducing an extraneous term using, for example, that if $a \geqslant b$ then $a+b=a$. Then the modular law is applied and then previously established relations are applied. Justification is usually given for steps which are not part of the above scheme.

1. Von Neumann $n$-frames. Let $L$ be a modular lattice. We say that $L$ contains an $n$-frame if there exist $a_{1}, \ldots, a_{n}, c_{12}, c_{13}, \ldots, c_{1 n} \in L$ such that (i) the sublattice generated by $a_{1}, \ldots, a_{n}$ is the Boolean algebra $2^{n}$ with 
atoms $a_{1}, \ldots, a_{n}$, and (ii) $a_{1}+c_{1 j}=a_{j}+c_{1 j}=a_{1}+a_{j}$ and $a_{1} c_{1 j}=a_{j} c_{1 j}=$ $a_{1} a_{j}$. In this situation we shall simply say that $\left\{a_{i}, c_{1 j}\right\}$ is an $n$-frame in $L$. We also allow the one element lattice to be a 'degenerate' $n$-frame. We let 0 denote the least element of this Boolean algebra, i.e., $0=a_{1} a_{2}$ and we do not insist that 0 is the least element of $L$. We let $P(n)$ denote an $n$-frame as a system of elements and relations and we let $F M(P(n))$ be the modular lattice freely generated by $a_{1}, \ldots, a_{n}, c_{12}, \ldots, c_{1 n}$ subject to the relations described above which make $\left\{a_{1}, \ldots, a_{n}, c_{12}, \ldots, c_{1 n}\right\}$ an $n$-frame. A great deal of information about $n$-frames is contained in [19], [15], [10], [11], [12].

EXAMPLE. Let $R$ be a ring with 1 and let ${ }_{R} R^{n}$ be the $n$-fold direct product of $n$ copies of $R$ as a left $R$-module. Let $L\left({ }_{R} R^{n}\right)$ be its lattice of submodules. Let

$$
\begin{gathered}
a_{i}=\{(0, \ldots, 0, x, 0, \ldots, 0): x \in R\}=R(0, \ldots, 0,1,0, \ldots, 0), \\
c_{i j}=R(0, \ldots,-1, \ldots, 1, \ldots, 0) \in L\left({ }_{R} R^{n}\right) .
\end{gathered}
$$

One easily checks that $\left\{a_{i}, c_{1 j}\right\}$ is an $n$-frame in $L\left({ }_{R} R^{n}\right)$ and that the $c_{i j}$ satisfy (1.1) below.

Let $\left\{a_{i}, c_{1 j} ; i=1, \ldots, n, j=2, \ldots, n\right\}$ be an $n$-frame in a modular lattice $L$. Let $c_{j 1}=c_{1 j}$ and for $1, i, j$ distinct let $c_{i j}=\left(c_{1 i}+c_{1 j}\right)\left(a_{i}+a_{j}\right)$. In Lemma 5.3 of [19, p. 118], it is shown that, for distinct $i, j, k$,

$$
c_{i k}=\left(c_{i j}+c_{j k}\right)\left(a_{i}+a_{k}\right) .
$$

In the definition of an $n$-frame the index 1 plays a special role. However, by (1.1), we see that this apparent lack of symmetry is only illusionary.

There are several concepts closely related to $n$-frames (cf. [10]). For example, a modular lattice is said to contain an $(n-1)$-diamond if it contains $2^{n}$ with atoms $a_{1}, \ldots, a_{n}$ and an element $y$ which is a relative complement of each $a_{i}$ in $a_{1}+\cdots+a_{n} / a_{1} a_{2}$. This concept was introduced by A. Huhn [11]. If $\left\{a_{1}, \ldots, a_{n}, y\right\}$ is an $(n-1)$-diamond in a modular lattice $L$, then $\left\{a_{i}, c_{1 j}\right\}$ is an $n$-frame, where $c_{1 j}=y\left(a_{1}+a_{j}\right)$. Conversely if $\left\{a_{i}, c_{1 j}\right\}$ is an $n$-frame in $L$ then $\left\{a_{i}, y\right\}$ is an $(n-1)$-diamond, where $y=\sum_{j=2}^{n} c_{1 j}[10]$.

LEMMA 1.1 (HERRMANN AND HUHN [10]). Let $\left\{a_{i}, c_{1 j}\right\}$ be an $n$-frame in $a$ modular lattice $L$. Let $b_{1} \in L$ satisfy $a_{1} a_{2}=0 \leqslant b_{1} \leqslant a_{1}$. Define $b_{i}=\left(b_{1}+\right.$ $\left.c_{1 i}\right) a_{i}$ and $b=\sum_{i=1}^{n} b_{i}$. Then $\left\{a_{i}^{\prime}, c_{1 j}^{\prime}\right\}$ and $\left\{a_{i}^{\prime \prime}, c_{1 j}^{\prime \prime}\right\}$ are both $n$-frames in $L$ where $a_{i}^{\prime}=a_{i}+b, c_{1 j}^{\prime}=c_{1 j}+b, a_{i}^{\prime \prime}=b_{i}$, and $c_{1 j}^{\prime \prime}=b c_{1 j}$. Moreover, in the primed frame, $c_{i j}^{\prime}$, which is defined to be $\left(c_{1 i}^{\prime}+c_{1 j}^{\prime}\right)\left(a_{i}^{\prime}+a_{j}^{\prime}\right)$, is equal to $c_{i j}+b$. Similarly $c_{i j}^{\prime \prime}=b c_{i j}$. 
Proof. Using modularity and $b_{k}<a_{k}$ and the independence of the $a_{i}$, we have

$$
\begin{aligned}
\left(a_{i}+b\right) \sum_{\substack{j=1 \\
j \neq i}}^{n}\left(a_{j}+b\right) & =b+a_{i}\left(\sum_{j \neq i} a_{j}+b\right) \\
& =b+a_{i}\left(\sum_{j \neq i} a_{j}+b_{i}\right) \\
& =b+b_{i}+a_{i}\left(\sum_{j \neq i} a_{j}\right) \\
& =b .
\end{aligned}
$$

Hence $\left\{a_{i}+b: i=1, \ldots, n\right\}$ are independent over $b$ and thus generate $2^{n}$. Now notice that, for $i \neq 1$,

$$
\begin{aligned}
b_{i}+c_{1 i} & =c_{1 i}+a_{i}\left(b_{1}+c_{1 i}\right) \\
& =\left(c_{1 i}+a_{i}\right)\left(b_{1}+c_{1 i}\right) \\
& =\left(c_{1 i}+a_{i}\right) b_{1}+c_{1 i} \\
& =\left(a_{1}+a_{i}\right) b_{1}+c_{1 i} \\
& =b_{1}+c_{1 i} .
\end{aligned}
$$

Using (1.2) we obtain the following for $i, j, 1$ distinct:

$$
\begin{aligned}
b_{i}+c_{i j} & =b_{i}+\left(c_{1 i}+c_{1 j}\right)\left(a_{i}+a_{j}\right) \\
& =\left(b_{i}+c_{1 i}+c_{1 j}\right)\left(a_{i}+a_{j}\right) \\
& =\left(b_{1}+c_{1 i}+c_{1 j}\right)\left(a_{i}+a_{j}\right) \\
& =\left(b_{j}+c_{1 i}+c_{1 j}\right)\left(a_{i}+a_{j}\right) \\
& =b_{j}+c_{i j} .
\end{aligned}
$$

For $i \neq 1$ we have

$$
\begin{aligned}
\left(a_{i}+b\right)\left(c_{1 i}+b\right) & =b+a_{i}\left(c_{1 i}+b\right) \\
& =b+a_{i}\left(a_{1}+a_{i}\right)\left(c_{1 i}+b_{1}+b_{i}+\sum_{j \neq 1, i} b_{j}\right) \\
& =b+a_{i}\left(c_{1 i}+b_{1}+b_{i}+\left(a_{1}+a_{i}\right) \sum_{j \neq 1, i} b_{j}\right) \\
& =b+b_{i}+a_{i}\left(c_{1 i}+b_{1}\right) \\
& =b+b_{i}+b_{i}=b .
\end{aligned}
$$


Using (1.2) we have

$$
\begin{aligned}
\left(a_{1}+b\right)\left(c_{1 i}+b\right) & =b+a_{1}\left(c_{1 i}+b_{1}+b_{i}\right) \\
& =b+a_{1}\left(c_{1 i}+b_{1}\right) \\
& =b+b_{1}+a_{1} c_{1 i}=b .
\end{aligned}
$$

Clearly $a_{1}^{\prime}+c_{1 j}^{\prime}=a_{j}^{\prime}+c_{1 j}^{\prime}=a_{1}^{\prime}+a_{j}^{\prime}$ and thus $\left\{a_{i}^{\prime}, c_{1 j}^{\prime}\right\}$ is an $n$-frame. It is also clear that $\left\{a_{i}^{\prime \prime}\right\}=\left\{b_{i}\right\}$ is independent over 0 , and calculations similar to those above complete the proof that $\left\{a_{i}^{\prime \prime}, c_{1 j}^{\prime \prime}\right\}$ is an $n$-frame.

Let $i, j, 1$ be distinct. Then by definition $c_{i j}^{\prime}=\left(c_{1 j}^{\prime}+c_{1 j}^{\prime}\right)\left(a_{i}^{\prime}+a_{j}^{\prime}\right)$. By (1.2), $b_{1} \leqslant b_{i}+c_{1 i}$. Thus

$$
\begin{aligned}
c_{i j}^{\prime}= & \left(c_{1 i}+c_{1 j}+b\right)\left(a_{i}+a_{j}+b\right) \\
& =b+\left(c_{1 i}+c_{1 j}+b\right)\left(a_{i}+a_{j}\right) \\
& =b+\left(c_{1 i}+c_{1 j}+\sum_{k=1}^{n} b_{k}\right)\left(a_{1}+a_{i}+a_{j}\right)\left(a_{i}+a_{j}\right) \\
& =b+\left(c_{1 i}+c_{1 j}+b_{1}+b_{i}+b_{j}+\left(\sum_{k \neq 1, i, j} b_{k}\right)\left(a_{1}+a_{i}+a_{j}\right)\right)\left(a_{i}+a_{j}\right) \\
& =b+\left(c_{1 i}+c_{1 j}+b_{i}+b_{j}\right)\left(a_{i}+a_{j}\right) \\
& =b+b_{i}+b_{j}+\left(c_{1 i}+c_{1 j}\right)\left(a_{i}+a_{j}\right) \\
& =b+c_{i j} .
\end{aligned}
$$

Similar calculations show that $c_{i j}^{\prime \prime}=b c_{i j}$, completing the proof.

Let $\left\{a_{i}, c_{1 j}\right\}$ be an $n$-frame in a modular lattice $L, n \geqslant 3$. Then for $i, j, k$ distinct we have the following projectivity:

$$
\begin{array}{r}
a_{i}+a_{j} / 0 \nearrow a_{i}+a_{j}+a_{k} / a_{k} \searrow c_{i k}+a_{j} / 0 \\
\nearrow a_{i}+a_{j}+a_{k} / c_{j k} \searrow a_{i}+a_{j} / 0 .
\end{array}
$$

This projectivity defines an automorphism $\alpha_{i j}$ of $a_{i}+a_{j} / 0$ given by

$$
\alpha_{i j}(x)=\left(\left(x+a_{k}\right)\left(c_{i k}+a_{j}\right)+c_{j k}\right)\left(a_{i}+a_{j}\right) .
$$

The above formula is a special case of von Neumann's addition defined on p. 142 of [19]. In fact, $\alpha_{i j}(x)$ corresponds to "adding one" to $x$. By Lemma 7.8 of [19], $\alpha_{i j}$ is independent of the choice of $k$ distinct from $i$ and $j$.

Let $b_{i}, i=1, \ldots, n$, and $b$ be the elements of $L$ given in Lemma 1.1 and let $\left\{a_{i}^{\prime}, c_{1 j}^{\prime}\right\}$ be the frame given by that lemma. Let $\alpha_{i j}^{\prime}$ be the automorphism corresponding to $\alpha_{i j}$ using the primed frame; that is

$$
\alpha_{i j}^{\prime}(x)=\left(\left(x+a_{k}^{\prime}\right)\left(c_{i k}^{\prime}+a_{j}^{\prime}\right)+c_{j k}^{\prime}\right)\left(a_{i}^{\prime}+a_{j}^{\prime}\right) .
$$

Let $0^{\prime}=b$. 
LEMMA 1.2. If $x \in a_{i}+a_{j} / 0$, then $x+b \in a_{i}^{\prime}+a_{j}^{\prime} / 0^{\prime}$ and $\alpha_{i j}^{\prime}(x+b)=$ $\alpha_{i j}(x)+b$.

Proof. First we show for distinct $i, j, k$ that

$$
\left(x+a_{k}+b\right)\left(c_{i k}+a_{j}+b\right)=b+\left(x+a_{k}\right)\left(c_{i k}+a_{j}\right) \text {. }
$$

Using $b_{i} \leqslant b_{k}+c_{i k}$ we calculate

$$
\begin{aligned}
\left(x+a_{k}+b\right)\left(c_{i k}+a_{j}+b\right) & =b+\left(x+a_{k}+b\right)\left(c_{i k}+a_{j}\right) \\
& =b+\left(x+a_{k}+b\right)\left(a_{i}+a_{j}+a_{k}\right)\left(c_{i k}+a_{j}\right) \\
& =b+\left(x+a_{k}+b_{i}+b_{j}\right)\left(c_{i k}+a_{j}\right) \\
& =b+\left(x+a_{k}+b_{i}\right)\left(c_{i k}+a_{j}\right)+b_{j} \\
& =b+\left(x+a_{k}+b_{i}\right)\left(c_{i k}+b_{k}+a_{j}\right)\left(c_{i k}+a_{j}\right) \\
& =b+\left(b_{i}+\left(x+a_{k}\right)\left(c_{i k}+b_{k}+a_{j}\right)\right)\left(c_{i k}+a_{j}\right) \\
& =b+\left(b_{i}+b_{k}+\left(x+a_{k}\right)\left(c_{i k}+a_{j}\right)\right)\left(c_{i k}+a_{j}\right) \\
& =b+\left(b_{i}+b_{k}\right)\left(c_{i k}+a_{j}\right)+\left(x+a_{k}\right)\left(c_{i k}+a_{j}\right) \\
& =b+\left(x+a_{k}\right)\left(c_{i k}+a_{j}\right) .
\end{aligned}
$$

Using this and $b_{k} \leqslant b_{j}+c_{j k}$ and letting $w=\left(x+a_{k}\right)\left(c_{i k}+a_{j}\right)$ we see that

$$
\begin{aligned}
\alpha_{i j}^{\prime}(x+b) & =\left[\left(x+a_{k}+b\right)\left(c_{i k}+a_{j}+b\right)+c_{j k}+b\right]\left(a_{i}+a_{j}+b\right) \\
& =\left[\left(x+a_{k}\right)\left(c_{i k}+a_{j}\right)+c_{j k}+b\right]\left(a_{i}+a_{j}+b\right) \\
& =b+\left[\left(x+a_{k}\right)\left(c_{i k}+a_{j}\right)+c_{j k}\right]\left(a_{i}+a_{j}+b\right) \\
& =b+\left(w+c_{j k}\right)\left(a_{i}+a_{j}+a_{k}\right)\left(a_{i}+a_{j}+b\right) \\
& =b+\left(w+c_{j k}\right)\left(a_{i}+a_{j}+b_{k}\right) \\
& =b+\left(w+c_{j k}\right)\left(w+c_{j k}+b_{j}\right)\left(a_{i}+a_{j}+b_{k}\right) \\
& =b+\left(w+c_{j k}\right)\left[b_{k}+\left(a_{i}+a_{j}\right)\left(w+c_{j k}+b_{j}\right)\right] \\
& =b+\left(w+c_{j k}\right)\left[b_{k}+b_{j}+\left(a_{i}+a_{j}\right)\left(w+c_{j k}\right)\right] \\
& =b+\left(w+c_{j k}\right)\left(b_{k}+b_{j}\right)+\left(a_{i}+a_{j}\right)\left(w+c_{j k}\right) \\
& =b+\left(a_{i}+a_{j}\right)\left(w+c_{j k}\right) \\
& =b+\alpha_{i j}(x) . \square
\end{aligned}
$$

Again maintaining the notation of Lemma 1.1, we let $\alpha_{i j}^{\prime \prime}$ be the automorphism of $a_{i}^{\prime \prime}+a_{j}^{\prime \prime} / 0$ obtained from $\alpha_{i j}$ by doubly priming everything.

LEMMA 1.3. If $x \in a_{i}+a_{j} / 0$, then $x b \in a_{i}^{\prime \prime}+a_{j}^{\prime \prime} / 0$ and $\alpha_{i j}^{\prime \prime}(x b)=b \alpha_{i j}(x)$. 
Proof. First note that

$$
x b=x\left(a_{i}+a_{j}\right) b=x\left(b_{i}+b_{j}\right)<b_{i}+b_{j}=a_{i}^{\prime \prime}+a_{j}^{\prime \prime} .
$$

Now

$$
\begin{aligned}
b\left(c_{i k}+a_{j}\right) & =\left(b_{i}+b_{j}+b_{k}\right)\left(c_{i k}+a_{j}\right) \\
& =b_{j}+\left(b_{i}+b_{k}\right)\left(a_{i}+a_{k}\right)\left(c_{i k}+a_{j}\right) \\
& =b_{j}+\left(b_{i}+b_{k}\right)\left(c_{i k}+\left(a_{i}+a_{k}\right) a_{j}\right) \\
& =b_{j}+\left(b_{i}+b_{k}\right) c_{i k} \\
& =b_{j}+b c_{i k} \\
& =a_{j}^{\prime \prime}+c_{i k}^{\prime \prime} .
\end{aligned}
$$

Furthermore

$$
\begin{aligned}
b\left(x+a_{k}\right) & =\left(b_{i}+b_{j}+b_{k}\right)\left(x+a_{k}\right) \\
& =b_{k}+\left(b_{i}+b_{j}\right)\left(a_{i}+a_{j}\right)\left(x+a_{k}\right) \\
& =b_{k}+\left(b_{i}+b_{j}\right) x \\
& =b_{k}+b x \\
& =b x+a_{k}^{\prime \prime} .
\end{aligned}
$$

Let $w=\left(x+a_{k}\right)\left(c_{i k}+a_{j}\right)$ as before. Since $b_{j}+b_{k}=b_{j}+b c_{j k}, w<a_{j}+c_{i k}$, and $c_{j k}\left(a_{j}+c_{i k}\right)=0$, we have

$$
\begin{aligned}
b\left(w+c_{j k}\right) & =\left(b_{i}+b_{j}+b_{k}\right)\left(w+c_{j k}\right) \\
& =\left(b c_{i k}+b_{j}+b c_{j k}\right)\left(w+c_{j k}\right) \\
& =b c_{j k}+\left(b c_{i k}+b_{j}\right)\left(w+c_{j k}\right) \\
& =b c_{j k}+\left(b c_{i k}+b_{j}\right)\left(a_{j}+c_{i k}\right)\left(w+c_{j k}\right) \\
& =b c_{j k}+\left(b_{j}+b c_{i k}\right) w \\
& \leqslant b c_{j k}+b w \\
& \leqslant b\left(w+c_{j k}\right) .
\end{aligned}
$$

Hence we have equality all the way across. Thus

$$
\begin{aligned}
\alpha_{i j}^{\prime \prime}(x b) & =\left(\left(x b+b_{k}\right)\left(b c_{i k}+b_{j}\right)+b c_{j k}\right)\left(b_{i}+b_{j}\right) \\
& =b\left(\left(x+a_{k}\right)\left(c_{i k}+a_{j}\right)+c_{j k}\right) b\left(a_{i}+a_{j}\right) \\
& =b \alpha_{i j}(x) .
\end{aligned}
$$

LEMMA 1.4. $\alpha_{i j}\left(a_{i}\right)=c_{i j}$ and $\alpha_{i j}\left(a_{j}\right)=a_{j}$. Consequently, if $x$ is a relative complement of $a_{j}$ in $a_{i}+a_{j} / 0$, then so is $\alpha_{i j}(x)$. 
Proof. This follows from easy calculations.

Let $\left\{a_{i}, c_{1 j}: i=1, \ldots, n, j=2, \ldots, n\right\}$ be an $n$-frame in a modular lattice $L, n \geqslant 3$, and let $r$ be a natural number. We say that $\left\{a_{i}, c_{1 j}\right\}$ is an $n$-frame of characteristic $r$ if $\alpha_{12}^{r}\left(a_{1}\right)=a_{1}$ where $\alpha_{12}$ is given in (1.5) and $\alpha_{12}^{r}$ is $\alpha_{12}$ iterated $r$ times.

EXAMPLE. In $L=L\left({ }_{R} R^{n}\right)$ it is easy to see that the submodules $R(-1, t, 0, \ldots, 0)$ correspond in a one-to-one fashion to the elements of $R$. By elementary linear algebra, $\alpha_{12}(R(-1, t, 0, \ldots, 0))=R(-1, t+$ $1,0, \ldots, 0)$. Since $a_{1}=R(-1,0, \ldots, 0)$, the equation $\alpha_{12}^{r}\left(a_{1}\right)=a_{1}$ holds in $L$ if and only if $r$ divides the characteristic of $R$.

Now let $\left\{a_{i}, c_{1 j}\right\}$ be an $n$-frame in an arbitrary modular lattice $L$ and let $r$ be a natural number. Let $b_{2}=a_{2}\left(\alpha_{12}^{r}\left(a_{1}\right)+a_{1}\right)$ and let

$$
b_{1}=a_{1}\left(b_{2}+c_{12}\right), \quad b_{i}=a_{i}\left(b_{1}+c_{1 i}\right), \quad i=3, \ldots, n \text {. }
$$

Note that $b_{2}=a_{2}\left(b_{1}+c_{12}\right)$ as

$$
\begin{aligned}
a_{2}\left(b_{1}+c_{12}\right) & =a_{2}\left(a_{1}\left(b_{2}+c_{12}\right)+c_{12}\right) \\
& =a_{2}\left(a_{1}+c_{12}\right)\left(b_{2}+c_{12}\right)=a_{2}\left(b_{2}+c_{12}\right)=b_{2} .
\end{aligned}
$$

Let $b=\sum_{i=1}^{n} b_{i}$.

LEMMA 1.5. Let $\left\{a_{i}^{\prime}, c_{1 j}^{\prime}\right\}=\left\{a_{i}+b, c_{1 j}+b\right\}$ be the $n$-frame given by Lemma 1.1 using the $b$ defined above. Let $\alpha_{12}^{\prime}$ be the automorphism (1.5) obtained by using the frame $\left\{a_{i}^{\prime}, c_{1 j}^{\prime}\right\}$. Then

$$
\left(\alpha_{12}^{\prime}\right)^{r}\left(a_{i}^{\prime}\right)=a_{1}^{\prime} .
$$

Proof. By Lemma 1.2

$$
\begin{aligned}
\left(\alpha_{12}^{\prime}\right)^{r}\left(a_{1}+b\right) & =\alpha_{12}^{r}\left(a_{1}\right)+b \\
& =\alpha_{12}^{r}\left(a_{1}\right)+b+a_{2}\left(\alpha_{12}^{r}\left(a_{1}\right)+a_{1}\right) \\
& =\left(\alpha_{12}^{r}\left(a_{1}\right)+a_{2}\right)\left(\alpha_{12}^{r}\left(a_{1}\right)+a_{1}\right)+b \\
& =\left(a_{1}+a_{2}\right)\left(\alpha_{12}^{r}\left(a_{1}\right)+a_{1}\right)+b \\
& =\alpha_{12}^{r}\left(a_{1}\right)+a_{1}+b
\end{aligned}
$$

since $\alpha_{12}^{r}\left(a_{1}\right)+a_{2}=a_{1}+a_{2}$ by Lemma 1.4. However,

$$
a_{1}+b_{2}=\left(a_{1}+a_{2}\right)\left(a_{1}+\alpha_{12}^{r}\left(a_{1}\right)\right)=a_{1}+\alpha_{12}^{r}\left(a_{1}\right) \text {. }
$$

Thus $a_{1}+b \geqslant \alpha_{12}^{r}\left(a_{1}\right)$. Hence $\left(\alpha_{12}^{\prime}\right)^{r}\left(a_{1}^{\prime}\right)=a_{1}+b=a_{1}^{\prime}$, as desired.

THEOREM 1.6. Let $M$ and $L$ be modular lattices and let $f$ be a homomorphism from $M$ onto $L$. If $\left\{a_{i}, c_{1 j}\right\}$ is an $n$-frame of characteristic $r$ in $L$, then there is an $n$-frame $\left\{\bar{a}_{i}, \bar{c}_{1 j}\right\}$ of characteristic $r$ in $M$ such that $f\left(\bar{a}_{i}\right)=a_{i}$ and $f\left(\bar{c}_{i j}\right)=c_{i j}$, $i, j=1, \ldots, n, i \neq j$. 
Proof. The corresponding result for $n$-frames (without regard to characteristic) was proved by András Huhn [11] (a proof is also given in [5]). Using this result we see that there is an $n$-frame $\left\{\bar{a}_{i}, \bar{c}_{1 j}\right\}$ in $M$ such that $f\left(\bar{a}_{i}\right)=a_{i}$ and $f\left(\bar{c}_{i j}\right)=c_{i j}$. Let $\bar{\alpha}_{12}$ be the automorphism of $\bar{a}_{1}+\bar{a}_{2} / \overline{0}$ given by (1.5) using the frame $\left\{\bar{a}_{i}, \bar{c}_{1 j}\right\}$. Define $\bar{b}_{2}=\bar{a}_{2}\left(\bar{\alpha}_{12}^{r}\left(\bar{a}_{1}\right)+\bar{a}_{1}\right)$ and

$$
\bar{b}_{1}=\bar{a}_{1}\left(\bar{b}_{2}+\bar{c}_{12}\right), \quad \bar{b}_{i}=\bar{a}_{i}\left(\bar{b}_{1}+\bar{c}_{1 i}\right), \quad i=3, \ldots, n, \quad \bar{b}=\sum_{i=1}^{n} \bar{b}_{i} .
$$

By Lemmas 1.1 and $1.5,\left\{\bar{a}_{i}+\bar{b}, \bar{c}_{1 j}+\bar{b}\right\}$ is an $n$-frame of characteristic $r$ in $M$. Since $\left\{a_{i}, c_{1 j}\right\}$ has characteristic $r$ by hypothesis,

$$
f\left(\bar{b}_{2}\right)=f\left(\left(\bar{\alpha}_{12}^{r}\left(\bar{a}_{1}\right)+\bar{a}_{1}\right) \bar{a}_{2}\right)=\left(\alpha_{12}^{r}\left(a_{1}\right)+a_{1}\right) a_{2}=\left(a_{1}+a_{1}\right) a_{2}=0 .
$$

Since $\overline{b_{i}} / \overline{0}$ is projective to $\bar{b}_{2} / \overline{0}$ in $M, f\left(\bar{b}_{i}\right)=0$. Thus $f(\bar{b})=0$. Consequently, $f\left(\bar{a}_{i}+\bar{b}\right)=a_{i}$ and $f\left(\bar{c}_{i j}+\bar{b}\right)=c_{i j}$, proving the theorem.

TheOREM 1.7. Let $\left\{a_{i}, c_{1 j}\right\}$ be an $n$-frame in a modular lattice L. Suppose $0 \leqslant b_{1}<c_{1} \leqslant a_{1}$. Let

$$
b_{i}=a_{i}\left(b_{1}+c_{1 i}\right), \quad c_{i}=a_{i}\left(c_{1}+c_{1 i}\right), \quad i=2, \ldots, n
$$

$b=\sum_{i=1}^{n} b_{i}, c=\sum_{i=1}^{n} c_{i}$. Then $\left\{a_{i} c+b, c_{1 j} c+b\right\}$ is also an n-frame. Moreover, if $\left\{a_{i}, c_{1 j}\right\}$ is an $n$-frame of characteristic $r$, then so is $\left\{a_{c} c+b, c_{1 j} c\right.$ $+b\}$. Furthermore

$$
\begin{aligned}
& a_{1}+a_{2} / 0 \supseteq c_{1}+c_{2} / b_{1}+b_{2} \nearrow c\left(a_{1}+b\right)+c\left(a_{2}+b\right) / b \\
& \nearrow c / \sum_{i=3}^{n} c\left(a_{i}+b\right) \nearrow c_{1}+c_{2}+\sum_{i=3}^{n} a_{i} / b_{1}+b_{2}+\sum_{i=3}^{n} a_{i} \subseteq 1 / \sum_{i=3}^{n} a_{i} .
\end{aligned}
$$

Proof. Since $\left(a_{i}+b\right) c=a_{i} c+b=c_{i}+b,\left(c_{i j}+b\right) c=c_{i j} c+b$, the first statement follows from two applications of Lemma 1.1. If $\left\{a_{i}, c_{1 j}\right\}$ has characteristic $r$, then so does $\left\{c a_{i}, c c_{1 j}\right\}$ by Lemma 1.3. By Lemma 1.2 $\left\{c a_{i}+b, c c_{1 j}+b\right\}$ has characteristic $r$ as well. Straightforward calculations establish the last part of the theorem.

2. The main result. Let $F$ and $K$ be countably infinite fields with char $F=$ $p$ and char $K=q$ for distinct primes $p$ and $q$. Let $L_{p}=L\left({ }_{F} F^{4}\right)$ be the lattice of subspaces of the $F$-vector space $F^{4}$. Similarly $L_{q}=L\left({ }_{K} K^{4}\right) . L_{p}$ contains the $n$-frame described in the example of $\S 1$. Namely, let $a_{i} \in L_{p}, i=$ $1,2,3,4$, be the subspace of vectors which are zero in every coordinate except possibly $i$. Let $c_{i j}, i, j=1,2,3,4, i \neq j$, be the subspace of those vectors which are zero in all coordinates except $i$ and $j$ and whose coordinates sum to zero. Thus $c_{12}$ is the subspace generated by $(-1,1,0,0)$. Let $a_{i}^{\prime}$, $c_{i j}^{\prime}$ be the corresponding elements in $L_{q}$. It is easy to see that $\left\{a_{i}, c_{1 j}\right\}$ is a 4-frame of characteristic $p$ and $\left\{a_{i}^{\prime}, c_{1 j}^{\prime}\right\}$ is a 4-frame of characteristic $q$. Since $|F|=|K|$ $=\omega$, the two-dimensional quotient sublattices $a_{1}+a_{2}+a_{3}+a_{4} / a_{3}+a_{4}$ of 
$L_{p}$ and $a_{1}^{\prime}+a_{2}^{\prime} / 0^{\prime}$ of $L_{q}$ are both isomorphic to $M_{\omega}$ (the countable twodimensional lattice). Let $\sigma$ be a lattice isomorphism from $a_{1}+a_{2}+a_{3}+$ $a_{4} / a_{3}+a_{4}$ onto $a_{1}^{\prime}+a_{2}^{\prime} / 0^{\prime}$ such that

$$
\begin{aligned}
\sigma\left(a_{1}+a_{2}+a_{3}+a_{4}\right) & =a_{1}^{\prime}+a_{2}^{\prime}, \\
\sigma\left(a_{3}+a_{4}\right) & =0^{\prime}, \\
\sigma\left(a_{1}+a_{3}+a_{4}\right) & =a_{1}^{\prime}, \\
\sigma\left(a_{2}+a_{3}+a_{4}\right) & =a_{2}^{\prime}, \\
\sigma\left(c_{12}+a_{3}+a_{4}\right) & =c_{12}^{\prime} .
\end{aligned}
$$

(The first two equations must hold.) Our lattice $L$ is defined on the disjoint union of $L_{p}$ and $L_{q}$. The order on $L$ is the transitive closure of the order on $L_{p}$, the order on $L_{q}$, and the order $x<\sigma(x), x \in a_{1}+a_{2}+a_{3}+a_{4} / a_{3}+a_{4}$. It is easy to see that $x<y$ holds in $L$ if and only if $x$ and $y$ are both in $L_{p}$ or $L_{q}$ and $x<y$ holds there, or $x \in L_{p}, y \in L_{q}$ and there is a $z \in a_{1}+a_{2}+a_{3}$ $+a_{4} / a_{3}+a_{4}$ such that $x \leqslant z$ and $\sigma(z) \leqslant y$. In $L$ we let

$$
\begin{aligned}
1=a_{1}+a_{2}+a_{3}+a_{4}, & & 1^{\prime}=a_{1}^{\prime}+a_{2}^{\prime}+a_{3}^{\prime}+a_{4}^{\prime}, \\
0^{\prime}=a_{1}^{\prime} a_{2}^{\prime}, & 0 & =a_{1} a_{2} .
\end{aligned}
$$

Note that 0 is the least element of $L$ and $1^{\prime}$ is the greatest. Also note that $1 / 0=L_{p}$ and $1^{\prime} / 0^{\prime}=L_{q}$ in $L$. Let $\theta$ be the congruence generated by $\left\{(x, \sigma(x)): x \in 1 / a_{3}+a_{4}\right\} . L / \theta$ is the lattice obtained from $L$ by identifying $x$ and $\sigma(x)$, i.e., $L / \theta$ is the lattice obtained from $L_{p}$ and $L_{q}$ using the usual Hall-Dilworth construction. The reader can check that $L / \theta$ is a simple lattice of length six. Moreover $L$ is a subdirect product of $L / \theta$ and the two element lattice. Thus, once we have shown that $L$ is not in the variety generated by finite modular lattices, it will follow that $L / \theta$ is also not in this variety.

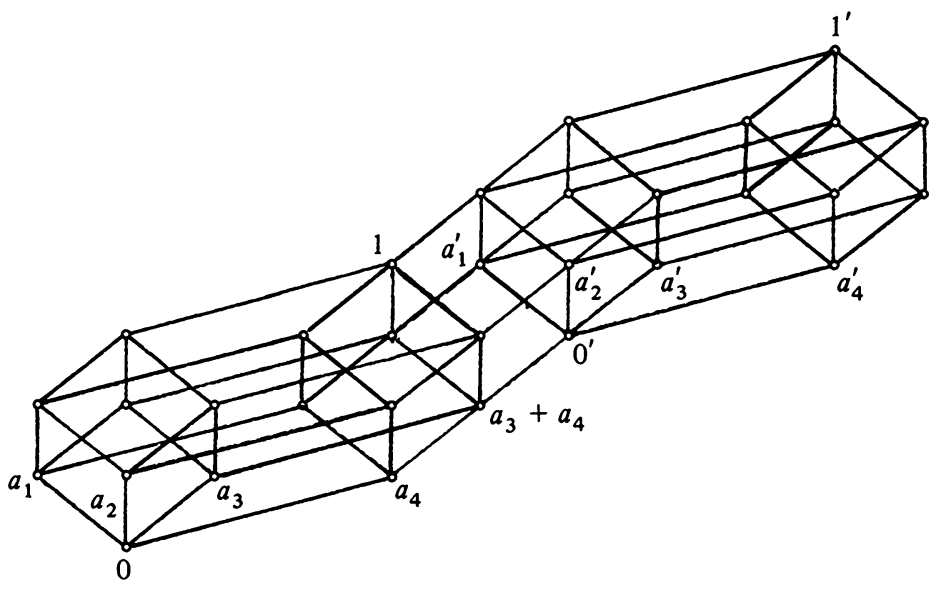

FiguRE 1 
The sublattice of $L$ generated by $\left\{a_{i}, a_{1}^{\prime}: i=1,2,3,4\right\}$ is a finite distributive lattice $D$, diagrammed in Figure 1 .

Notice that $1 / a_{3}+a_{4} \nearrow a_{1}^{\prime}+a_{2}^{\prime} / 0^{\prime}$.

THEOREM 2.1. $L$ is a modular lattice not in the variety generated by all finite modular lattices.

Proof. $L$ is a modular lattice by [4] and [9]. Let $\mathfrak{K}_{f}$ be the class of all finite modular lattices. By Birkhoff's theorem the variety generated by $\mathfrak{R}_{f}$ is $H S P\left(\Re_{f}\right)$, the class of all homomorphic images of sublattices of direct products of lattices in $\mathfrak{N}_{f}$. If $L \in H S P\left(\Re_{f}\right)$, then there would be an $M \in S P\left(\Re_{f}\right)$ and a homomorphism from $M$ onto $L$. We will show that this is impossible by showing that whenever $M$ is a modular lattice mapping homomorphically onto $L$ then $M$ is not residually finite (= a subdirect product of finite lattices). Of course, $M \in S P\left(\mathscr{N}_{f}\right)$ implies $M$ is residually finite.

Thus we suppose $M$ is a modular lattice and $f$ is a homomorphism from $M$ onto $L$. Most of this section will be occupied in showing that a great deal of the structure of $L$ can be pulled back through $f$ into $M$. To begin with we note that the sublattice $D$ of $L$ diagrammed in Figure 1 is a projective modular lattice. This follows from Mitschke's and Wille's result that a finite distributive lattice is a projective modular lattice if the join-irreducible elements are closed under meets and the partially ordered set of these elements contains no crown [17]. Alternately $D$ is a direct product of the four element Boolean algebra and the lattice diagrammed in Figure 2.

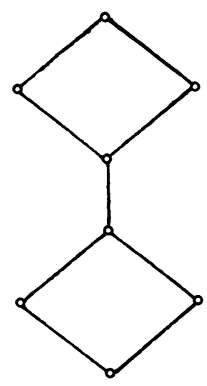

FIGURE 2

It is easy to see that both these lattices are projective modular lattices and that the class of finite projective modular lattices is closed under finite direct products [5]. Hence there exist $\bar{a}_{i}$ and $\bar{a}_{i}^{\prime}$ in $M, i=1,2,3,4$, which generate a sublattice isomorphic to $D$ and such that $f\left(\bar{a}_{i}\right)=a_{i}$ and $f\left(\bar{a}_{i}^{\prime}\right)=a_{i}^{\prime}$. In $L_{q} \subseteq L$ we let $e^{\prime}=\sum_{i \neq j} c_{i j}^{\prime}$. Notice that

$$
e^{\prime}=\left\{\left(v_{1}, v_{2}, v_{3}, v_{4}\right) \in K^{4}: v_{1}+v_{2}+v_{3}+v_{4}=0\right\}
$$


and that $e^{\prime}$ is a relative complement of $a_{i}^{\prime}$ in $a_{1}^{\prime}+a_{2}^{\prime}+a_{3}^{\prime}+a_{4}^{\prime} / 0^{\prime}, i=$ $1, \ldots, 4$, and that $c_{i j}^{\prime}=e^{\prime}\left(a_{i}^{\prime}+a_{j}^{\prime}\right), i \neq j$. Choose $\bar{e}^{\prime} \in M$ such that

$$
\overline{0}^{\prime}=\bar{a}_{1}^{\prime} \bar{a}_{2}^{\prime} \leqslant \bar{e}^{\prime} \leqslant \bar{a}_{1}^{\prime}+\bar{a}_{2}^{\prime}+\bar{a}_{3}^{\prime}+\bar{a}_{4}^{\prime}
$$

and $f\left(\bar{e}^{\prime}\right)=e^{\prime}$. Set $\bar{b}^{\prime}=\sum_{i=1}^{4} \bar{a}_{i}^{\prime} \bar{e}^{\prime}, \bar{c}=\prod_{i=1}^{4}\left(\bar{a}_{i}^{\prime}+\bar{e}^{\prime}\right)$, and

$$
\bar{d}^{\prime}=\sum_{i=1}^{4}\left(\bar{a}_{i}^{\prime}+\bar{b}^{\prime}\right) \bar{c}^{\prime}=\overline{b^{\prime}}+\sum_{i=1}^{4} \bar{a}_{i}^{\prime} \bar{c}^{\prime} .
$$

It is shown in [5] using straightforward calculations that $\left\{\bar{a}_{i}^{\prime} \bar{c}^{\prime}+\bar{b}^{\prime}, 1 \leqslant i \leqslant\right.$ 4) generates a copy of $2^{4}$ with $\bar{b}^{\prime}$ and $\bar{d}^{\prime}$ as least and greatest elements respectively. Since $a_{i}^{\prime} e^{\prime}=0^{\prime}$ in $L, f\left(\bar{a}_{i}^{\prime} \bar{e}^{\prime}\right)=0^{\prime}$ and thus $f\left(\bar{b}^{\prime}\right)=0^{\prime}$. Similarly $f\left(\bar{c}^{\prime}\right)=1^{\prime}$ and hence $f\left(\bar{a}_{i}^{\prime} \bar{c}^{\prime}+\bar{b}^{\prime}\right)=a_{i}^{\prime}, i=1,2,3,4$. Moreover, it is shown that $\bar{e}^{\prime} \bar{d}^{\prime}$ is a relative complement of each $\bar{a}_{i}^{\prime} \bar{c}^{\prime}+\bar{b}^{\prime}$ in $\bar{d}^{\prime} / \bar{b}^{\prime}$. Furthermore, $f\left(\bar{e}^{\prime} \bar{d}^{\prime}\right)=e^{\prime}$. Hence if we define $\bar{c}_{1 j}^{\prime}=\left(\bar{a}_{1}^{\prime} \bar{c}+\bar{a}_{j}^{\prime} \bar{c}^{\prime}+\bar{b}^{\prime}\right) \bar{e}^{\prime} \bar{d}^{\prime}$, then it is easy to calculate that $f\left(\bar{c}_{1 j}^{\prime}\right)=c_{1 j}^{\prime}$ and that $\bar{c}_{1 j}^{\prime}$ is a relative complement of $\bar{a}_{1}^{\prime} \bar{c}^{\prime}+b^{\prime}$ and $\bar{a}_{j}^{\prime} \bar{c}^{\prime}+\bar{b}^{\prime}$ in $\bar{a}_{i}^{\prime} \bar{c}^{\prime}+\bar{a}_{j}^{\prime} \bar{c}^{\prime}+\bar{b}^{\prime} / \bar{b}^{\prime}$. Consequently $\left\{\bar{a}_{i}^{\prime} \bar{c}^{\prime}+\bar{b}^{\prime}\right\} \cup\left\{\bar{c}_{1}^{\prime}\right\}$ forms a 4-frame mapping to $\left\{a_{i}^{\prime}, c_{1 j}^{\prime}\right\}$ under $f$.

Now consider the elements $\bar{a}_{1} d^{\prime}+\left(\bar{a}_{1}+\bar{a}_{2}\right) \bar{b}^{\prime}, \bar{a}_{2} \bar{d}^{\prime}+\left(\bar{a}_{1}+\bar{a}_{2}\right) \bar{b}^{\prime}, \bar{a}_{3}+\left(\bar{a}_{1}\right.$ $\left.+\bar{a}_{2}\right) \bar{b}^{\prime}, \bar{a}_{4}+\left(\bar{a}_{1}+\bar{a}_{2}\right) \bar{b}^{\prime}$. Since $f\left(\overline{b^{\prime}}\right)=0$, these four elements map under $f$ to $a_{1}, a_{2}, a_{3}, a_{4}$, respectively. Since $\bar{d}^{\prime}<\bar{c}^{\prime}, \bar{a}_{3}+\bar{a}_{4}<\overline{0}^{\prime}<\bar{b}^{\prime}, \bar{a}_{1}<\bar{a}_{1}^{\prime}, \bar{a}_{2}<$ $\bar{a}_{2}^{\prime}$, and $\left\{\bar{a}_{i}^{\prime} \bar{c}^{\prime}+\bar{b}^{\prime}, \bar{c}_{1 j}^{\prime}\right\}$ is a 4-frame, we have

$$
\begin{gathered}
\left(\bar{a}_{1} \bar{d}^{\prime}+\left(\bar{a}_{1}+\bar{a}_{2}\right) \bar{b}^{\prime}\right)\left(\bar{a}_{2} \bar{d}^{\prime}+\bar{a}_{3}+\bar{a}_{4}+\left(\bar{a}_{1}+\bar{a}_{2}\right) \bar{b}^{\prime}\right) \\
\leqslant\left(\bar{a}_{1}^{\prime} \bar{c}^{\prime}+\bar{b}^{\prime}\right)\left(\bar{a}_{2}^{\prime} \bar{c}^{\prime}+\bar{b}^{\prime}\right)=\bar{b}^{\prime} .
\end{gathered}
$$

Since the left-hand side is less than or equal to $\bar{a}_{1}+\bar{a}_{2}$, it is less than or equal to $\left(\bar{a}_{1}+\bar{a}_{2}\right) \bar{b}^{\prime}$. But the other inequality is obvious. Now

$$
\begin{aligned}
\left(\bar{a}_{3}+\right. & \left.\left(\bar{a}_{1}+\bar{a}_{2}\right) \bar{b}^{\prime}\right)\left(\bar{a}_{1} \bar{d}^{\prime}+\bar{a}_{2} \bar{d}^{\prime}+\bar{a}_{4}+\left(\bar{a}_{1}+\bar{a}_{2}\right) \bar{b}^{\prime}\right) \\
& =\left(\bar{a}_{1}+\bar{a}_{2}\right) \bar{b}^{\prime}+\bar{a}_{3}\left(\bar{a}_{1} \bar{d}^{\prime}+\bar{a}_{2} \bar{d}^{\prime}+\bar{a}_{4}+\left(\bar{a}_{1}+\bar{a}_{2}\right) \bar{b}^{\prime}\right) \\
& =\left(\bar{a}_{1}+\bar{a}_{2}\right) \bar{b}^{\prime},
\end{aligned}
$$

since $\bar{a}_{1} \bar{d}^{\prime}+\bar{a}_{2} \bar{d}^{\prime}+\bar{a}_{4}+\left(\bar{a}_{1}+\bar{a}_{2}\right) \bar{b}^{\prime} \leqslant \bar{a}_{1}+\bar{a}_{2}+\bar{a}_{4}$. These calculations show that $\bar{a}_{1} \bar{d}^{\prime}+\left(\bar{a}_{1}+\bar{a}_{2}\right) \bar{b}^{\prime}, \bar{a}_{2} \bar{d}^{\prime}+\left(\bar{a}_{1}+\bar{a}_{2}\right) \bar{b}^{\prime}, \bar{a}_{3}+\left(\bar{a}_{1}+\bar{a}_{2}\right) \bar{b}^{\prime}, \bar{a}_{4}+\left(\bar{a}_{1}\right.$ $\left.+\bar{a}_{2}\right) \bar{b}^{\prime}$ are independent over $\left(\bar{a}_{1}+\bar{a}_{2}\right) \bar{b}^{\prime}$, and hence generate a copy of $2^{4}$. Moreover,

$$
\left(\bar{a}_{1} \bar{d}^{\prime}+\bar{a}_{2} \bar{d}^{\prime}+\bar{a}_{3}+\bar{a}_{4}+\left(\bar{a}_{1}+\bar{a}_{2}\right) \bar{b}^{\prime}\right)+\bar{b}^{\prime}=\bar{a}_{1}^{\prime} \bar{d}^{\prime}+\bar{a}_{2}^{\prime} \bar{d}^{\prime}+\bar{b}^{\prime}
$$

Clearly the right-hand side is larger, but

$$
\bar{a}_{1} \bar{d}^{\prime}+\bar{b}^{\prime} \geqslant \bar{a}_{1} \bar{d}^{\prime}+\overline{0}^{\prime}=\left(\bar{a}_{1}+\overline{0}^{\prime}\right) \bar{d}^{\prime}=\bar{a}_{1}^{\prime} \bar{d}^{\prime},
$$


from which the opposite inclusion follows. Now

$$
\begin{gathered}
\left(\bar{a}_{1} \bar{d}^{\prime}+\bar{a}_{2} \bar{d}^{\prime}+\bar{a}_{3}+\bar{a}_{4}+\left(\bar{a}_{1}+\bar{a}_{2}\right) \bar{b}^{\prime}\right) \bar{b}^{\prime} \\
=\bar{a}_{3}+\bar{a}_{4}+\left(\bar{a}_{1}+\bar{a}_{2}\right) \bar{b}^{\prime}+\left(\bar{a}_{1} \bar{d}^{\prime}+\bar{a}_{2} \bar{d}^{\prime}\right) \bar{b}^{\prime} \\
=\bar{a}_{3}+\bar{a}_{4}+\left(\bar{a}_{1}+\bar{a}_{2}\right) \bar{b}^{\prime} .
\end{gathered}
$$

Consequently,

$$
\begin{gathered}
\bar{a}_{1} \bar{d}^{\prime}+\bar{a}_{2} \bar{d}^{\prime}+\bar{a}_{3}+\bar{a}_{4}+\left(\bar{a}_{1}+\bar{a}_{2}\right) \bar{b}^{\prime} / \bar{a}_{3}+\bar{a}_{4}+\left(\bar{a}_{1}+\bar{a}_{2}\right) \overline{b^{\prime}} \\
\nearrow \bar{a}_{1}^{\prime} \bar{d}^{\prime}+\bar{a}_{2}^{\prime} \overline{d^{\prime}}+\overline{b^{\prime}} / \overline{b^{\prime}}=\bar{a}_{1}^{\prime} \bar{c}^{\prime}+\bar{a}_{2}^{\prime} \bar{c}^{\prime}+\overline{b^{\prime}} / \overline{b^{\prime}} .
\end{gathered}
$$

Here we have used $\bar{a}_{1}^{\prime} \bar{c}^{\prime}=\bar{a}_{1}^{\prime} \bar{d}^{\prime}$ which follows easily from the definitions (cf. [5]). It now follows that the sublattice of $M$ generated by $\left\{\bar{a}_{i}^{\prime} \bar{c}^{\prime}+\bar{b}^{\prime}\right\} \cup$ $\left\{\bar{a}_{1} \bar{d}^{\prime}+\left(\bar{a}_{1}+\bar{a}_{2}\right) \bar{b}^{\prime}, \bar{a}_{2} \bar{d}^{\prime}+\left(\bar{a}_{1}+\bar{a}_{2}\right) \bar{b}^{\prime}, \bar{a}_{3}+\left(\bar{a}_{1}+\bar{a}_{2}\right) \bar{b}^{\prime}, \bar{a}_{4}+\left(\bar{a}_{1}+\bar{a}_{2}\right) \bar{b}^{\prime}\right\}$ is the lattice $D$ diagrammed in Figure 1 .

Thus, changing notation, we see that there exist $\left\{\bar{a}_{i}\right\} \cup\left\{\bar{a}_{i}^{\prime}\right\} \cup\left\{\bar{c}_{i j}^{\prime}\right\} \subseteq M$ such that

$$
f\left(\bar{a}_{i}\right)=\bar{a}_{i}, \quad f\left(\bar{a}_{i}^{\prime}\right)=\bar{a}_{i}^{\prime}, \quad f\left(\bar{c}_{i j}^{\prime}\right)=\bar{c}_{i j}^{\prime},
$$

and $\left\{a_{i}^{\prime}, c_{1 j}^{\prime}\right\}$ is a 4-frame, and $\left\{\bar{a}_{i}, \bar{a}_{i}^{\prime}\right\}$ generates $D$. Let $\bar{\alpha}_{12}^{\prime}$ be the automorphism of $\bar{a}_{1}^{\prime}+\bar{a}_{2}^{\prime} / \overline{0}^{\prime}$ given by (1.5) relative to the frame $\left\{\bar{a}_{i}^{\prime}, \bar{c}_{1 j}^{\prime}\right\}$. Let $\bar{b}_{2}^{\prime}=\bar{a}_{2}^{\prime}\left(\bar{a}_{1}^{\prime}+\alpha_{12}^{\prime q}\left(\bar{a}_{1}^{\prime}\right)\right)$ and

$$
\overline{b_{1}^{\prime}}=\bar{a}_{1}^{\prime}\left(\bar{b}_{2}^{\prime}+\bar{c}_{12}^{\prime}\right), \quad \overline{b_{i}^{\prime}}=\bar{a}_{i}^{\prime}\left(\bar{b}_{1}^{\prime}+\bar{c}_{1 i}^{\prime}\right), \quad \overline{b^{\prime}}=\sum_{i=1}^{4} \overline{b_{i}^{\prime}} .
$$

Then by Lemma $1.5\left\{\bar{a}_{i}^{\prime}+\bar{b}^{\prime}, \bar{c}_{1 j}^{\prime}+\bar{b}^{\prime}\right\}$ forms a 4-frame of characteristic $q$. Since the frame $\left\{a_{1}^{\prime}, c_{1 j}^{\prime}\right\}$ in $L$ has characteristic $q$, it follows that $f\left(\bar{b}^{\prime}\right)=0^{\prime}$ and thus

$$
f\left(\bar{a}_{i}^{\prime}+\bar{b}^{\prime}\right)=a_{i}^{\prime}, \quad f\left(\bar{c}_{i j}^{\prime}+\bar{b}^{\prime}\right)=c_{i j}^{\prime} .
$$

Moreover $f\left(\bar{a}_{i}+\left(\bar{a}_{1}+\bar{a}_{2}\right) \bar{b}^{\prime}\right)=a_{i}, i=1,2,3,4$, and calculations similar to the above show that $\left\{\bar{a}_{i}+\left(\bar{a}_{1}+\bar{a}_{2}\right) \bar{b}^{\prime}\right\} \cup\left\{\bar{a}_{i}^{\prime}+\bar{b}^{\prime}\right\}$ generates a sublattice isomorphic to $D$.

Changing notation again we see that there exist $\bar{a}_{i}^{\prime}, \bar{c}_{i j}^{\prime}, \bar{a}_{i}$ such that their images under $f$ are $a_{i}^{\prime}, c_{i j}^{\prime}, a_{i}$ and $\left\{\bar{a}_{i}^{\prime}, \bar{c}_{1 j}^{\prime}\right\}$ is a 4-frame of characteristic $q$, and $\left\{\bar{a}_{i}^{\prime}, \bar{a}_{i}\right\}$ generates a copy of $D$.

Since $c_{23}+c_{24} \leqslant a_{2}+a_{3}+a_{4}$, it is possible to choose $\overline{\bar{e}} \in M$ such that $f(\overline{\bar{e}})=c_{23}+c_{34}$ and $\overline{0} \leqslant \bar{e} \leqslant \bar{a}_{2}+\bar{a}_{3}+\bar{a}_{4}$. Let $\overline{\bar{c}}_{12}=\bar{c}_{12}^{\prime}\left(\bar{a}_{1}+\bar{a}_{2}\right)$ and notice that $f\left(\bar{c}_{12}\right)=c_{12}$ since $c_{12}^{\prime}\left(a_{1}+a_{2}\right)=c_{12}$ by the construction of $L$. Let $\bar{e}=\overline{\bar{c}}_{12}+\bar{e}$. Let $\bar{b}=\sum_{i=1}^{4} \bar{a}_{i} \bar{e}, \bar{c}=\prod_{i=1}^{4}\left(\bar{a}_{i}+\bar{e}\right)$ and 


$$
\begin{aligned}
\bar{d} & =\sum_{i=1}^{4}\left(\bar{a}_{i}+\bar{b}\right) \bar{c}=\sum_{i=1}^{4} \bar{a}_{i} \bar{c}, \\
\bar{c}_{i j} & =\left(\bar{a}_{i} \bar{c}+\bar{a}_{j} \bar{c}+\bar{b}\right) \bar{e} \bar{d}=\left(\bar{a}_{i} \bar{c}+\bar{a}_{j} \bar{c}+\bar{b}\right) \bar{e}=\left(\bar{a}_{i} \bar{c}+\bar{a}_{j} \bar{c}\right) \bar{e}+\bar{b} .
\end{aligned}
$$

As before $\left\{\bar{a}_{i} \bar{c}+\bar{b}, \bar{c}_{1 j}\right\}$ is a 4-frame mapping to $\left\{a_{i}, c_{1 j}\right\}$ under $f$. Easy calculations show that

$$
\begin{aligned}
\bar{a}_{1} \bar{c}+\bar{a}_{2} \bar{c}+\bar{b} / \bar{b} \lambda \bar{d} / \bar{a}_{3} \bar{c}+\bar{a}_{4} \bar{c}+\bar{b} & \nearrow \bar{d}+\bar{o}^{\prime} / \bar{b}+\bar{o}^{\prime} \\
& =\bar{a}_{1} \bar{c}+\bar{a}_{2} \bar{c}+\overline{0}^{\prime} / \bar{a}_{1} \bar{e}+\bar{a}_{2} \bar{e}+\bar{o}^{\prime} \\
& \subseteq \bar{a}_{1}^{\prime}+\bar{a}_{2}^{\prime} / \bar{o}^{\prime} .
\end{aligned}
$$

This last quotient is illustrated in Figure 3.

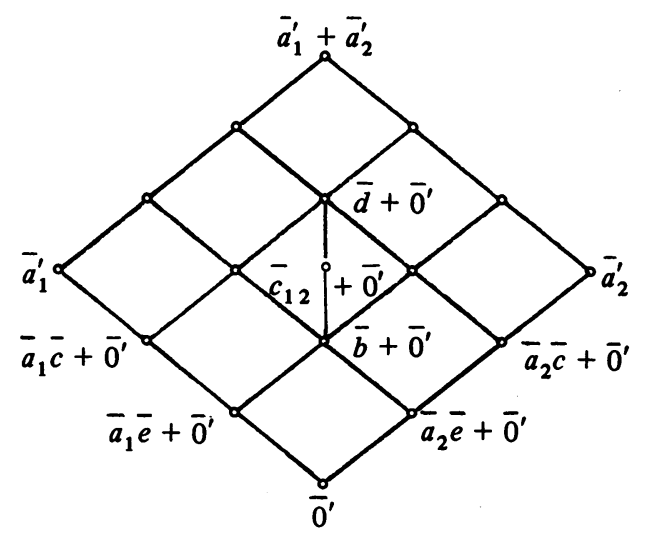

Figure 3

Note that

$$
\overline{\bar{c}}_{12}+\overline{0}^{\prime}=\bar{c}_{12}^{\prime}\left(\bar{a}_{1}+\bar{a}_{2}\right)+0^{\prime}=\bar{c}_{12}^{\prime}\left(\bar{a}_{1}+\bar{a}_{2}+\overline{0}^{\prime}\right)=\bar{c}_{12}^{\prime}\left(\bar{a}_{1}^{\prime}+\bar{a}_{2}^{\prime}\right)=\bar{c}_{12}^{\prime} \text {. }
$$

Also

$$
\begin{aligned}
\bar{a}_{1}+\overline{\bar{c}}_{12} & =\bar{a}_{1}+\bar{c}_{12}^{\prime}\left(\bar{a}_{1}+\bar{a}_{2}\right)=\left(\bar{a}_{1}+\bar{c}_{12}^{\prime}\right)\left(\bar{a}_{1}+\bar{a}_{2}\right) \\
& =\left(\bar{a}_{1}+\overline{0}^{\prime}+\bar{c}_{12}^{\prime}\right)\left(\bar{a}_{1}+\bar{a}_{2}\right)=\left(\bar{a}_{1}^{\prime}+\bar{a}_{2}^{\prime}\right)\left(\bar{a}_{1}+\bar{a}_{2}\right)=\bar{a}_{1}+\bar{a}_{2} .
\end{aligned}
$$

Similarly $\bar{a}_{2}+\overline{\bar{c}}_{12}=\bar{a}_{1}+\bar{a}_{2}$ and $\bar{a}_{1} \overline{\bar{c}}_{12}=\overline{0}=\bar{a}_{2} \overline{\bar{c}}_{12}$. Now since $\overline{\bar{c}}_{12}<\bar{e}$,

$$
\overline{\bar{c}}_{12}+\bar{a}_{1} \bar{e}=\left(\overline{\bar{c}}_{12}+\bar{a}_{1}\right) \bar{e}=\left(\overline{\bar{c}}_{12}+\bar{a}_{2}\right) \bar{e}=\overline{\bar{c}}_{12}+\bar{a}_{2} \bar{e}
$$

Similarly, since $\overline{\bar{c}}_{12}<\bar{e}<\bar{c}$,

$$
\overline{\bar{c}}_{12}+\bar{a}_{1} \bar{c}=\overline{\bar{c}}_{12}+\bar{a}_{2} \bar{c}
$$


Define $\bar{b}_{1}^{\prime}=\bar{a}_{1} \bar{e}+\overline{0}^{\prime}, \bar{b}_{i}^{\prime}=\bar{a}_{i}^{\prime}\left(\bar{b}_{1}^{\prime}+\bar{c}_{1 i}^{\prime}\right), \bar{c}_{1}^{\prime}=\bar{a}_{1} \bar{c}+\overline{0}^{\prime}, \bar{c}_{i}^{\prime}=\bar{a}_{i}^{\prime}\left(\bar{c}_{1}^{\prime}+\bar{c}_{1 i}^{\prime}\right)$. Then, since $\bar{c}_{12}^{\prime}=\overline{0}^{\prime}+\overline{\bar{c}}_{12}$

$$
\begin{aligned}
\bar{b}_{2}^{\prime} & =\bar{a}_{2}^{\prime}\left(\bar{a}_{1} \bar{e}+\overline{0}^{\prime}+\bar{c}_{12}^{\prime}\right)=\bar{a}_{2}^{\prime}\left(\bar{a}_{1} \bar{e}+\overline{0}^{\prime}+\overline{\bar{c}}_{12}\right) \\
& =\bar{a}_{2}^{\prime}\left(\bar{a}_{2} \bar{e}+\overline{0}^{\prime}+\bar{c}_{12}\right)=\bar{a}_{2}^{\prime}\left(\bar{a}_{2} \bar{e}+\bar{c}_{12}^{\prime}\right) \\
& =\bar{a}_{2} \bar{e}+\bar{a}_{2}^{\prime} \bar{c}_{12}^{\prime}=\bar{a}_{2} \bar{e}+\overline{0}^{\prime} .
\end{aligned}
$$

Similarly

$$
\bar{c}_{2}^{\prime}=\bar{a}_{2} \bar{c}+\overline{0}^{\prime}
$$

Let $\overline{b^{\prime}}=\overline{b_{1}^{\prime}}+\overline{b_{2}^{\prime}}+\overline{b_{3}^{\prime}}+\overline{b_{4}^{\prime}}, \bar{c}^{\prime}=\bar{c}_{1}^{\prime}+\bar{c}_{2}^{\prime}+\bar{c}_{3}^{\prime}+\bar{c}_{4}^{\prime}$. By Theorem $1.7\left\{\bar{a}_{i}^{\prime} \bar{c}^{\prime}\right.$ $\left.+\bar{b}^{\prime}, \bar{c}_{1 j}^{\prime} \bar{c}^{\prime}+\bar{b}^{\prime}\right\}$ is a 4 -frame of characteristic $q$. Moreover

$$
\begin{gathered}
\bar{d} / \bar{a}_{3} \bar{c}+\bar{a}_{4} \bar{c}+\bar{b} \lambda \bar{a}_{1}^{\prime} \bar{c}^{\prime}+\bar{a}_{2}^{\prime} \bar{c}^{\prime}+\bar{b}^{\prime} / \bar{b}^{\prime}, \\
\left(\bar{a}_{i} \bar{c}+\bar{b}\right)+\bar{b}^{\prime}=\bar{a}_{i}^{\prime} \bar{c}^{\prime}+\bar{b}^{\prime}, \quad i=1,2, \\
\bar{c}_{12}+\bar{b}^{\prime}=\bar{c}_{12}^{\prime} \bar{c}^{\prime}+\bar{b}^{\prime} .
\end{gathered}
$$

To see these first note that

$$
\bar{a}_{1}^{\prime} \bar{c}^{\prime}=\bar{a}_{1}^{\prime}\left(\bar{a}_{1} \bar{c}+\bar{a}_{2} \bar{c}+\overline{0}^{\prime}+\bar{c}_{3}^{\prime}+\bar{c}_{4}^{\prime}\right)=\bar{a}_{1} \bar{c}+\overline{0}^{\prime},
$$

since $\bar{a}_{2} \bar{c}+\bar{c}_{\underline{3}}^{\prime}+\bar{c}_{4}^{\prime} \leqslant \bar{a}_{2}^{\prime}+\bar{a}_{3}^{\prime}+\bar{a}_{4}^{\prime}$. Similarly $\bar{a}_{2}^{\prime} \bar{c}^{\prime}=\bar{a}_{2} \bar{c}+\overline{0}^{\prime}$. Thus, since $\bar{a}_{3} \bar{c}+\bar{a}_{4} \bar{c} \leqslant \bar{b}^{\prime}$

$$
\begin{aligned}
\bar{d}+\bar{b}^{\prime} & =\sum_{i=1}^{4} \bar{a}_{i} \bar{c}+\bar{b}^{\prime}=\sum_{i=1}^{4} \bar{a}_{i} \bar{c}+\overline{0}^{\prime}+\bar{b}^{\prime} \\
& =\bar{a}_{1}^{\prime} \bar{c}^{\prime}+\bar{a}_{2}^{\prime} \bar{c}^{\prime}+\bar{b}^{\prime}, \\
\bar{d} \cdot \bar{b}^{\prime} & =\bar{d}\left(\bar{a}_{1}^{\prime}+\bar{a}_{2}^{\prime}\right)\left(\bar{a}_{1} \bar{e}+\bar{a}_{2} \bar{e}+\overline{0}^{\prime}+\bar{b}_{3}^{\prime}+\bar{b}_{4}\right) \\
& =\bar{d}\left(\bar{a}_{1} \bar{e}+\bar{a}_{2} \bar{e}+\overline{0}^{\prime}+\left(\bar{a}_{1}^{\prime}+\bar{a}_{2}^{\prime}\right)\left(\bar{b}_{3}^{\prime}+\bar{b}_{4}^{\prime}\right)\right) \\
& =\bar{d}\left(\bar{a}_{1} \bar{e}+\bar{a}_{2} \bar{e}+\overline{0}^{\prime}\right) \\
& =\bar{a}_{1} \bar{e}+\bar{a}_{2} \bar{e}+\left(\bar{a}_{1} \bar{c}+\bar{a}_{2} \bar{c}+\bar{a}_{3} \bar{c}+\bar{a}_{4} \bar{c}\right) \overline{0}^{\prime} \\
& =\bar{a}_{1} \bar{e}+\bar{a}_{2} \bar{e}+\left(\bar{a}_{1} \bar{c}+\bar{a}_{2} \bar{c}\right) \overline{0}^{\prime}+\bar{a}_{3} \bar{c}+\bar{a}_{4} \bar{c} \\
& =\bar{a}_{3} \bar{c}+\bar{a}_{4} \bar{c}+\bar{b} .
\end{aligned}
$$

Since $\bar{a}_{i} \bar{c}+\bar{b}+\bar{b}^{\prime}=\bar{a}_{i} \bar{c}+\overline{0}^{\prime}+\bar{b}^{\prime}=\bar{a}_{i}^{\prime} \bar{c}^{\prime}+\bar{b}^{\prime}$, (2.3) holds. Now, since $\overline{\bar{e}} \leqslant \bar{a}_{2}+\bar{a}_{3}+\bar{a}_{4}$ and $\overline{\bar{e}} \leqslant \bar{e} \leqslant \bar{c}$, 


$$
\begin{aligned}
\bar{c}_{12} & =\left(\bar{a}_{1} \bar{c}+\bar{a}_{2} \bar{c}\right) \bar{e}+\bar{b} \\
& =\left(\bar{a}_{1} \bar{c}+\bar{a}_{2} \bar{c}\right)\left(\bar{a}_{1}+\bar{a}_{2}\right)\left(\overline{\bar{c}}_{12}+\overline{\bar{e}}\right)+\bar{b} \\
& =\left(\bar{a}_{1} \bar{c}+\bar{a}_{2} \bar{c}\right)\left(\overline{\bar{c}}_{12}+\left(\bar{a}_{1}+\bar{a}_{2}\right) \overline{\bar{e}}\right)+\bar{b} \\
& =\left(\bar{a}_{1} \bar{c}+\bar{a}_{2} \bar{c}\right)\left(\overline{\bar{c}}_{12}+\bar{a}_{2} \overline{\bar{e}}\right)+\bar{b} \\
& =\overline{\bar{c}}_{12}\left(\bar{a}_{1} \bar{c}+\bar{a}_{2} \bar{c}\right)+\bar{a}_{2} \overline{\bar{e}}+\bar{b} \\
& \leqslant \bar{c}_{12}^{\prime} \bar{c}^{\prime}+\bar{b}^{\prime} .
\end{aligned}
$$

In the last step we have used $\overline{\bar{c}}_{12} \leqslant \bar{c}_{12}^{\prime}, \bar{a}_{1} \bar{c}+\bar{a}_{2} \bar{c} \leqslant \bar{c}^{\prime}$, and $\bar{a}_{2} \overline{\bar{e}}<\bar{b}<\bar{b}^{\prime}$. Thus $\bar{c}_{12}+\bar{b}^{\prime}<\bar{c}_{12}^{\prime} \bar{c}^{\prime}+\bar{b}^{\prime}$. However $\left\{\bar{a}_{1} \bar{c}+\bar{b}, \bar{a}_{2} \bar{c}+\bar{b}, \bar{c}_{12}\right\}$ and $\left\{\bar{a}_{1}^{\prime} \bar{c}^{\prime}+\right.$ $\left.\bar{b}, \bar{a}_{2}^{\prime} \bar{c}^{\prime}+\bar{b}^{\prime}, \bar{c}_{12}^{\prime} \bar{c}^{\prime}+\bar{b}^{\prime}\right\}$ generate $M_{3}$ (since they are each a part of a frame). Using (2.2) and (2.3) one can check that $\bar{a}_{1} \bar{c}+\bar{a}_{2} \bar{c}+\bar{b} / \bar{b} \nearrow \bar{a}_{1}^{\prime} \bar{c}^{\prime}+\bar{a}_{2}^{\prime} \bar{c}^{\prime}+$ $\overline{b^{\prime}} / \overline{b^{\prime}}$ with the image of $\bar{a}_{i} \bar{c}+\bar{b}$ equalling $\bar{a}_{i}^{\prime} \bar{c}+\bar{b}^{\prime}, i=1,2$. Thus $\bar{a}_{1}^{\prime} \bar{c}^{\prime}+\overline{b^{\prime}}$, $\bar{a}_{2}^{\prime} \bar{c}^{\prime}+\bar{b}^{\prime}, \bar{c}_{12}+\bar{b}^{\prime}$ also generates $M_{3}$. Since $\bar{c}_{12}+\bar{b}^{\prime}<\bar{c}_{12}^{\prime} \bar{c}^{\prime}+\bar{b}^{\prime}$ modularity implies they are equal, proving (2.4).

Thus changing notation again we see that there exist $\bar{a}_{i}, \bar{a}_{i}^{\prime}, \bar{c}_{i j}$, $\bar{c}_{i j}^{\prime}$ in $M$ with $f\left(\bar{a}_{i}\right)=a_{i}$, etc., $\left\{\bar{a}_{i}, \bar{c}_{1 j}\right\}$ a 4-frame, $\left\{\bar{a}_{i}^{\prime}, \bar{c}_{1 j}^{\prime}\right\}$ a 4-frame of characteristic $q$. Moreover, the sublattice generated by $\left\{a_{i}, a_{i}^{\prime}\right\}$ is $D$ by (2.2) and (2.3) and, by (2.4), $\bar{c}_{12}+\overline{0}^{\prime}=\bar{c}_{12}^{\prime}$.

Let $\bar{\alpha}_{12}$ be the automorphism of $\bar{a}_{1}+\bar{a}_{2} / \overline{0}$ given by (1.5) relative to the frame $\left\{\bar{a}_{i}, \bar{c}_{1 j}\right\}$. Let

$$
\bar{b}_{2}=\bar{a}_{2}\left(\bar{a}_{1}+\bar{\alpha}_{12}^{p}\left(\bar{a}_{1}\right)\right), \quad \bar{b}_{1}=\bar{a}_{1}\left(\bar{b}_{2}+\bar{c}_{12}\right), \quad \bar{b}_{i}=\bar{a}_{i}\left(\bar{b}_{1}+\bar{c}_{1 i}\right), \quad \bar{b}=\sum_{i=1}^{4} \bar{b}_{i}
$$

By Lemma $1.5\left\{\bar{a}_{i}+\bar{b}, \bar{c}_{1 j}+\bar{b}\right\}$ is a 4-frame of characteristic $p$. Moreover $f\left(\bar{a}_{i}+\bar{b}\right)=a_{i}$, etc. Let $\bar{b}_{1}^{\prime}=\bar{b}_{1}+\overline{0}^{\prime}, \bar{b}_{i}^{\prime}=\bar{a}_{i}^{\prime}\left(\bar{b}_{1}^{\prime}+\bar{c}_{1 i}^{\prime}\right)$, and $\overline{b^{\prime}}=\sum_{i=1}^{4} \overline{b_{i}^{\prime}}$. Since $\bar{b}_{2}+\bar{c}_{12}=\bar{b}_{1}+\bar{c}_{12}$, and $\bar{c}_{12}^{\prime}=\bar{c}_{12}+\overline{0}^{\prime}$

$$
\bar{b}_{1}^{\prime}+\bar{c}_{12}^{\prime}=\bar{b}_{1}+\overline{0}^{\prime}+\bar{c}_{12}=\bar{b}_{2}+\bar{c}_{12}+\overline{0}^{\prime}
$$

So that

$$
\begin{aligned}
\overline{b_{2}^{\prime}} & =\bar{a}_{2}^{\prime}\left(\overline{b_{1}^{\prime}}+\bar{c}_{12}^{\prime}\right)=\bar{a}_{2}^{\prime}\left(\bar{b}_{2}+\bar{c}_{12}+\overline{0}^{\prime}\right) \\
& =\bar{b}_{2}+\overline{0}^{\prime}+\bar{a}_{2}^{\prime} \bar{c}_{12}=\bar{b}_{2}+\overline{0}^{\prime} .
\end{aligned}
$$

By Theorem 1.7 (with $c_{1}=a_{1}$ ) $\left\{\bar{a}_{i}^{\prime}+\bar{b}^{\prime}, \bar{c}_{1 j}^{\prime}+\bar{b}^{\prime}\right\}$ is a 4-frame of characteristic $q$. Moreover

$$
\bar{a}_{1}+\bar{a}_{2}+\bar{a}_{3}+\bar{a}_{4} / \bar{a}_{3}+\bar{a}_{4}+\bar{b} \nearrow \bar{a}_{1}^{\prime}+\bar{a}_{2}^{\prime}+\bar{b}^{\prime} / \bar{b}^{\prime}
$$

and the image of $\bar{a}_{i}+\bar{a}_{3}+\bar{a}_{4}+\bar{b}$ under this map is $\bar{a}_{i}^{\prime}+\bar{b}^{\prime}, i=1,2$. To see this note that $\bar{a}_{1}+\bar{a}_{2}+\bar{a}_{3}+\bar{a}_{4}+\bar{b}^{\prime}=\bar{a}_{1}^{\prime}+\bar{a}_{2}^{\prime}+\bar{b}^{\prime}$, since $\overline{\sigma^{\prime}}<\bar{b}^{\prime}$ and $\bar{a}_{3}+$ $\bar{a}_{4}<\bar{b}^{\prime}$. Also 


$$
\begin{aligned}
\left(\bar{a}_{1}+\bar{a}_{2}+\bar{a}_{3}+\bar{a}_{4}\right) \bar{b}^{\prime} & =\left(\bar{a}_{1}+\bar{a}_{2}+\bar{a}_{3}+\bar{a}_{4}\right)\left(\bar{b}_{1}+\bar{b}_{2}+\bar{b}_{3}^{\prime}+\bar{b}_{4}^{\prime}\right) \\
& =\bar{b}_{1}+\bar{b}_{2}+\left(\bar{a}_{1}+\bar{a}_{2}+\bar{a}_{3}+\bar{a}_{4}\right)\left(\bar{a}_{3}^{\prime}+\bar{a}_{4}^{\prime}\right)\left(\bar{b}_{3}^{\prime}+\bar{b}_{4}^{\prime}\right) \\
& =\bar{b}_{1}+\bar{b}_{2}+\bar{a}_{3}+\bar{a}_{4}=\bar{a}_{3}+\bar{a}_{4}+\bar{b}
\end{aligned}
$$

Since $\bar{b}^{\prime} \geqslant \overline{0}^{\prime}$ and $\bar{a}_{i}+\overline{0}^{\prime}=\bar{a}_{i}^{\prime}, i=1,2$, we have

$$
\bar{a}_{i}+\bar{b}^{\prime}=\bar{a}_{i}^{\prime}+\bar{b}^{\prime}
$$

Thus with our final change in notation we have elements $\bar{a}_{i}, \bar{a}_{i}^{\prime}, \bar{c}_{i j}, \bar{c}_{i j}^{\prime}$ in $M$ such that $f\left(\bar{a}_{i}\right)=a_{i}$, etc., $\left\{\bar{a}_{i}, \bar{a}_{i}^{\prime}\right\}$ generates $D,\left\{\bar{a}_{i}, \bar{c}_{1 j}\right\}$ is a 4 -frame of characteristic $p$, and $\left\{\bar{a}_{i}^{\prime}, \bar{c}_{1 j}\right\}$ is a 4-frame of characteristic $q$.

We will now show that $M$ is not residually finite. This will be done with the aid of von Neumann coordinatization. We now review the results from [19] which we will require. In Part II of [19], von Neumann coordinatizes complemented modular lattices containing an $n$-frame with $n \geqslant 4$. In the first part of the proof he uses the lattice to construct a ring (the auxiliary ring) and shows that it is in fact a ring with 1 (cf. [19, pp. 130-157, especially Theorem 8.4]). This part of the proof does not use complementation. Thus associated with any $n$-frame, $n \geqslant 4$, in a modular lattice is an auxiliary ring.

Now suppose our lattice $M$ is residually finite. Since $\bar{a}_{i}, \bar{a}_{i}^{\prime}, \bar{c}_{1 j}, \bar{c}_{1 j}^{\prime}$ are inverse images of the cooresponding elements in $L$, they are distinct elements in $M$. Since there are only finitely many of them, there is a homomorphism $g$ mapping $M$ to a finite modular lattice $A$ which is one-to-one on the set $\left\{\bar{a}_{i}, \bar{a}_{i}^{\prime}, \bar{c}_{1 j}, \bar{c}_{1 j}^{\prime}: i=1,2,3,4, j=2,3,4\right\}$. Since $g$ is a homomorphism $\left\{g\left(\bar{a}_{i}\right), g\left(\bar{c}_{1 j}\right)\right\}$ is a 4-frame of characteristic $p$ in $A$. The auxiliary ring defined by this frame has for its underlying set

$$
R=\left\{x \in A: x+g\left(\bar{a}_{2}\right)=g\left(\bar{a}_{1}\right)+g\left(\bar{a}_{2}\right) \text { and } x g\left(\bar{a}_{2}\right)=g(\overline{0})\right\}
$$

(cf. Definition 6.1 and Lemma 6.1 of [19, p. 130], $L_{i j}$ is defined on p. 95 of [19]). If $x, y \in R$, then their sum in $R$, denoted here $x \oplus y$, is defined to be

$$
\begin{gathered}
{\left[\left(x+g\left(\bar{a}_{3}\right)\right)\left(g\left(\bar{a}_{2}\right)+g\left(\bar{c}_{13}\right)\right)+\left(y+g\left(\bar{c}_{13}\right)\right)\left(g\left(\bar{a}_{2}\right)+g\left(\bar{a}_{3}\right)\right)\right]} \\
\cdot\left(g\left(\bar{a}_{1}\right)+g\left(\bar{a}_{2}\right)\right)
\end{gathered}
$$

(cf. Definition 7.5 and Theorem 7.1 of [19]). The element $g\left(\bar{a}_{1}\right) \in R$ is the null element of $R$ and $g\left(\bar{c}_{12}\right) \in R$ is the unit of $R$. Since $\left(\bar{c}_{12}+\bar{c}_{13}\right)\left(\bar{a}_{2}+\bar{a}_{3}\right)$ $=\bar{c}_{23}$, we see upon comparing the addition in $R$ to the definition of $\alpha_{12}$ given in (1.5) that if $x \in R, \alpha_{12}(x)=x \oplus 1$. Since the frame $\left\{g\left(\bar{a}_{i}\right), g\left(\bar{c}_{1 j}\right)\right\}$ has characteristic $p, \alpha_{12}^{p}\left(g\left(\bar{a}_{1}\right)\right)=g\left(\bar{a}_{1}\right)$. This says that $R$ satisfies $0=0+1$ $+\cdots+1$ ( $p$ l's), i.e., $R$ has characteristic $p$. Since $A$ is finite, $R$ is finite by (2.5). This implies $|R|=p^{n}$, for some $n$, since the underlying group of $R$ is a $p$-group. Moreover, since $g\left(\bar{a}_{1}\right) \neq g\left(\bar{c}_{12}\right)$ are both in $R,|R|>2$. 
$\left\{g\left(\bar{a}_{i}^{\prime}\right), g\left(\bar{c}_{1 j}^{\prime}\right)\right\}$ is also a 4-frame in $A$. This frame determines a ring $R^{\prime}$ with underlying set

$$
R^{\prime}=\left\{x \in A: x+g\left(\bar{a}_{2}^{\prime}\right)=g\left(\bar{a}_{1}^{\prime}\right)+g\left(\bar{a}_{2}^{\prime}\right) \text { and } x g\left(\bar{a}_{2}^{\prime}\right)=g\left(\overline{0}^{\prime}\right)\right\} .
$$

Since $\left\{g\left(\bar{a}_{i}^{\prime}\right), g\left(\bar{c}_{1 j}^{\prime}\right)\right\}$ is a 4-frame of characteristic $q$, arguments as above show that $\left|R^{\prime}\right|=q^{m}$ for some $m$.

Since $\left\{\bar{a}_{i}, \bar{a}_{i}^{\prime}\right\}$ generates the sublattice $D$ in $M$, we have the transposition

$$
\bar{a}_{1}+\bar{a}_{2} / 0 \nearrow \bar{a}_{1}^{\prime}+\bar{a}_{2}^{\prime} / \overline{0}^{\prime} \text {. }
$$

Thus $\bar{a}_{1}+\bar{a}_{2} / \overline{0} \cong \bar{a}_{1}^{\prime}+\bar{a}_{2}^{\prime} / 0^{\prime}$ via the map $x \mapsto x+\overline{0}^{\prime}$. Note that $\bar{a}_{2} \mapsto \bar{a}_{2}^{\prime}$. Since $g$ is a homomorphism, $x \mapsto x+g\left(\overline{0}^{\prime}\right)$ is an isomorphism from $g\left(\bar{a}_{1}\right)+$ $g\left(\bar{a}_{2}\right) / g(\overline{0})$ onto $g\left(\bar{a}_{1}^{\prime}\right)+g\left(\bar{a}_{2}^{\prime}\right) / g\left(\overline{0}^{\prime}\right)$ which sends $g\left(\bar{a}_{2}\right)$ to $g\left(\bar{a}_{2}^{\prime}\right)$. Note that $R \subseteq g\left(\bar{a}_{1}\right)+g\left(\bar{a}_{2}\right) / g(\overline{0})$ and $R^{\prime} \subseteq g\left(\bar{a}^{\prime}\right)+g\left(\bar{a}^{\prime}\right) / g\left(\overline{0}^{\prime}\right)$. Checking the definition of $R$ and $R^{\prime}$ one sees that the above isomorphism restricted to $R$ is a bijection from $R$ onto $R^{\prime}$. Thus $p^{n}=|R|=\left|R^{\prime}\right|=q^{m}$, a contradiction since $|R|>2$. This completes the proof.

3. Further results. If $\mathfrak{V}$ is a variety of lattices, we let $F_{\curlyvee}(\kappa)$ denote the free $\checkmark$-algebra on $\kappa$ generators. $F L(\kappa), F M(\kappa)$ and $F D(\kappa)$ denote the free, free modular, and free distributive lattices, respectively. If $\kappa$ is infinite note that every proper quotient sublattice of $F_{\gamma}(\kappa)$ has cardinality $\kappa$. It is not hard to see that every proper quotient sublattice of $F L(\omega)$ satisfies no nontrivial lattice identities. The corresponding result for modular lattices is false. It is shown in [8] that if $M_{3}$ is a sublattice of a modular lattice $L$ then $a / b$ (see Figure 4) is Arguesian.

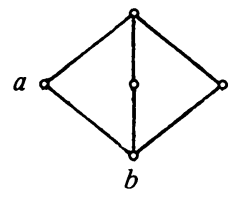

FIGURE 4

Hence $F M(\kappa)$ contains quotients satisfying the Arguesian identity. In this section we show that $F M(\kappa)$ has a proper distributive quotient and thus there are arbitrarily large distributive lattices which can be embedded into free modular lattices. This should be compared with the result of Galvin and Jónsson that there are no uncountable distributive lattices embeddable in free lattices. The above result has the corollary that $F D(\kappa)$ is embeddable into $F M(\kappa)$ for each infinite $\kappa$. To see this let $a / b$ be a proper distributive quotient of $F M(\kappa)$. Let $\kappa^{\prime}=\kappa-(\operatorname{var}(a) \cup \operatorname{var}(b))$. Then $\left|\kappa^{\prime}\right|=|\kappa|$ and it is not hard to prove that the sublattice generated by $\left\{a x+b: x \in \kappa^{\prime}\right\}$ is isomorphic to 
$F D(\kappa)$. (This also follows from a more general unpublished result of Joel Berman.) We mention one contrasting example. Let $\mathcal{V}$ be the variety generated by all subspace lattices of vector spaces over a fixed field $F$. Then the proper quotients of $F_{\Upsilon}(\omega)$ satisfy no identities other than those of $\mathfrak{V}$.

THEOREM 3.1. Let $\kappa$ be a cardinal, $\kappa \geqslant 5$. Then $F M(\kappa)$ contains a proper distributive quotient.

Proof. Let $L$ be the lattice constructed in $\S 2$. We shall see below that $L$ can be generated by five elements. Thus let $f$ be a homomorphism from $F M(\kappa)$ onto $L$. By the proof of Theorem 2.1, with $F M(\kappa)$ in place of $M$, $F M(\kappa)$ contains two 4-frames $\left\{a_{i}, c_{1 j}\right\}$ and $\left\{a_{i}^{\prime}, c_{1 j}^{\prime}\right\}$, the first of characteristic $p$, the second of characteristic $q$ such that the sublattice generated by $\left\{a_{i}, a_{i}^{\prime}\right.$ : $i=1,2,3,4\}$ is $D$ (see Figure 1) and $c_{12}^{\prime}=c_{12}+a_{1}^{\prime} a_{2}^{\prime}$. Here we have omitted the bars on $a_{i}$, etc. As before we let $0=a_{1} a_{2}$ and $0^{\prime}=a_{1}^{\prime} a_{2}^{\prime}$; but note that neither of these elements is the least element of $F M(\kappa)$. We shall show that $a_{1} / 0$ is distributive. If this is not the case, then $a_{1} / 0$ contains $M_{3}$ as a sublattice. We may assume that $a_{1}$ is the greatest element of $M_{3}$ and 0 is the least element. For if this is not the case then, using Theorem 1.7, we can adjust the elements $a_{i}, c_{1 j}, a_{i}^{\prime}, c_{1 j}^{\prime}$ so that it is true. This type of argument was given several times in $\$ 2$ and so will not be repeated here.

Let $b_{1}, b_{5}, d_{15}$ be the atoms of $M_{3}$. Thus $b_{1}+b_{5}=b_{1}+d_{15}=b_{5}+d_{15}=$ $a_{1}$ and $b_{1} b_{5}=b_{1} d_{15}=b_{5} d_{15}=0$. Define $b_{2}, \ldots, b_{8}$ as follows

$$
\begin{aligned}
& b_{i}=a_{i}\left(b_{1}+c_{1 i}\right), \quad i=2,3,4, \\
& b_{i}=a_{i-4}\left(b_{5}+c_{1 i-4}\right), \quad i=6,7,8 .
\end{aligned}
$$

It is easy to see that $b_{1}, \ldots, b_{8}$ are independent over 0 . Let $e=c_{12}+c_{13}+$ $c_{14}$ and let $e^{*}=e+d_{15}$. As was mentioned earlier, $e$ is a relative complement of $a_{i}, i=1,2,3,4$, in $a_{1}+a_{2}+a_{3}+a_{4} / 0$ and thus $e\left(a_{1}+a_{j}\right)=c_{1 j}$ (cf. [10]). From this one can show that $e^{*}$ is a relative complement of each $b_{j}$ in the same quotient. If we now define $d_{1 j}=e^{*}\left(b_{1}+b_{j}\right), i=2, \ldots, 8$, it is easy to see that $\left\{b_{i}, d_{1 j}: i=1, \ldots, 8, j=2, \ldots, 8\right\}$ is an 8 -frame. Furthermore, for $j=2,3,4, d_{1 j}=c_{1 j}\left(b_{1}+b_{j}\right)$. To see this note since $c_{1 j} \leqslant e \leqslant e^{*}, d_{1 j}=e^{*}\left(b_{1}\right.$ $\left.+b_{j}\right) \geqslant c_{1 j}\left(b_{1}+b_{j}\right)$. However using equation (3.1) one can check that $d_{1 j}$ and $c_{1 j}\left(b_{1}+b_{j}\right)$ are both relative complements of $b_{1}$ in $b_{1}+b_{j} / 0$. Now modularity yields $d_{1 j}=c_{1 j}\left(b_{1}+b_{j}\right)$. From this observation and Theorem 1.7 it follows that $\left\{b_{1}, b_{2}, b_{3}, b_{4}, d_{12}, d_{13}, d_{14}\right\}$ is a 4-frame of characteristic $p$, since $\left\{a_{i}, c_{1 j}\right\}$ has characteristic $p$.

By Theorem 5.1 of [19, p. 127], there is a projective isomorphism from $b_{1}+b_{2}+b_{3}+b_{4} / 0$ onto $b_{1}+b_{2}+b_{5}+b_{6} / 0=a_{1}+a_{2} / 0$ (as $b_{1}+b_{5}=$ $a_{1}, b_{2}+b_{6}=a_{2}$ ). Moreover this isomorphism maps $d_{12}$ to $d_{12}, d_{13}$ to $d_{15}$, and $d_{14}$ to $d_{16}$. Thus $\left\{b_{1}, b_{2}, b_{5}, b_{6}, d_{12}, d_{15} d_{16}\right\}$ is also a 4-frame of characteristic $p$. 
Since $a_{1} / 0 \lambda a_{1}^{\prime} / 0^{\prime}$, the elements $b_{1}^{\prime}=b_{1}+a_{1}^{\prime} a_{2}^{\prime}, b_{5}^{\prime}=b_{5}+a_{1}^{\prime} a_{2}^{\prime}, d_{15}^{\prime}=d_{15}$ $+a_{1}^{\prime} a_{2}^{\prime}$ together with $a_{1}^{\prime}$ and $0^{\prime}$ form a copy of $M_{3}$. Elements $b_{1}^{2}, \ldots, b_{8}^{\prime}, d_{12}^{\prime}, \ldots, d_{18}^{\prime}$ can be defined in a manner similar to $b_{1}, \ldots, b_{8}, d_{12}, \ldots, d_{18}$. Arguments similar to those given above show that $\left\{b_{1}^{\prime}, b_{2}^{\prime}, b_{5}^{\prime}, b_{6}^{\prime}, d_{12}^{\prime}, d_{15}^{\prime}, d_{16}^{\prime}\right\}$ is a 4-frame of characteristic $q$.

We claim that $b_{i}^{\prime}=b_{i}+0^{\prime}$ for $i=1,2,5,6$ and $d_{12}^{\prime}=d_{12}+0^{\prime}, d_{15}^{\prime}=d_{15}+$ $0^{\prime}$, and $d_{16}^{\prime}=d_{16}+0^{\prime}$. For $b_{1}^{\prime}, b_{5}^{\prime}$ and $d_{15}^{\prime}$ the above hold by definition. By definition $b_{6}^{\prime}=\left(b_{5}^{\prime}+c_{12}^{\prime}\right) a_{2}^{\prime}$. Thus, as $0=\left(a_{1}+a_{2}\right) 0^{\prime}$,

$$
\begin{aligned}
b_{6}^{\prime} & =\left(b_{5}+c_{12}+0^{\prime}\right)\left(a_{2}+0^{\prime}\right) \\
& =\left(b_{5}+c_{12}+0^{\prime}\right)\left(a_{1}+a_{2}\right) a_{2}+0^{\prime} \\
& =\left(b_{5}+c_{12}\right) a_{2}+0^{\prime} \\
& =b_{6}+0^{\prime} .
\end{aligned}
$$

Similarly $b_{2}^{\prime}=b_{2}+0^{\prime}$. To handle the remaining formulas we shall first prove that $d_{12}+d_{15}+d_{16}=c_{12}+d_{15}$. Indeed, since $b_{1}+e^{*}=a_{1}+a_{2}+a_{3}+a_{4}$, and $d_{15}+b_{1}=a_{1}, b_{2}+b_{6}=a_{2}$,

$$
\begin{aligned}
d_{15}+d_{12}+d_{16} & =d_{15}+e^{*}\left(b_{1}+b_{2}\right)+e^{*}\left(b_{1}+b_{6}\right) \\
& =d_{15}+e^{*}\left(b_{1}+b_{2}+e^{*}\left(b_{1}+b_{6}\right)\right) \\
& =d_{15}+e^{*}\left(b_{2}+\left(e^{*}+b_{1}\right)\left(b_{1}+b_{6}\right)\right) \\
& =d_{15}+\left(d_{15}+c_{12}+c_{13}+c_{14}\right)\left(b_{2}+b_{1}+b_{6}\right) \\
& =\left(d_{15}+c_{12}+c_{13}+c_{14}\right)\left(d_{15}+b_{2}+b_{1}+b_{6}\right) \\
& =\left(d_{15}+c_{12}+c_{13}+c_{14}\right)\left(a_{1}+a_{2}\right) \\
& =d_{15}+c_{12}+\left(c_{13}+c_{14}\right)\left(a_{1}+a_{2}\right) \\
& =d_{15}+c_{12} .
\end{aligned}
$$

(The reader can verify $\left(c_{13}+c_{14}\right)\left(a_{1}+a_{2}\right)=0$.) Now by definition

$$
\begin{aligned}
d_{16}^{\prime} & =\left(d_{15}^{\prime}+c_{12}^{\prime}+c_{13}^{\prime}+c_{14}^{\prime}\right)\left(b_{1}^{\prime}+b_{6}^{\prime}\right) \\
& =\left(d_{15}+c_{12}+c_{13}^{\prime}+c_{14}^{\prime}\right)\left(b_{1}+b_{6}\right)+0^{\prime} \\
& =\left(d_{15}+c_{12}+c_{13}^{\prime}+c_{14}^{\prime}\right)\left(a_{1}+a_{2}\right)\left(b_{1}+b_{6}\right)+0^{\prime} \\
& =\left(d_{15}+c_{12}\right)\left(b_{1}+b_{6}\right)+0^{\prime} \\
& =\left(d_{15}+d_{12}+d_{16}\right)\left(b_{1}+b_{6}\right)+0^{\prime} \\
& =d_{16}+0^{\prime} .
\end{aligned}
$$

Similarly, $d_{12}^{\prime}=d_{12}+0^{\prime}$.

We know that $a_{1}+a_{2} / 0>a_{1}^{\prime}+a_{2}^{\prime} / 0^{\prime}$. Thus $x \mapsto x+0$ is an isomorphism of these quotients. The above calculations show that this isomorphism sends the 4-frame $\left\{b_{1}, b_{2}, b_{5}, b_{6}, d_{12}, d_{15}, d_{16}\right\}$ onto the 4-frame $\left\{b_{1}^{\prime}, b_{2}^{\prime}, b_{5}^{\prime}, b_{6}^{\prime}, d_{12}^{\prime}, d_{15}^{\prime}, d_{16}^{\prime}\right\}$. It follows that the sublattices generated by these 
two 4-frames are isomorphic, and hence that $\left\{b_{1}, b_{2}, b_{5}, b_{6}, d_{12}, d_{15}, d_{16}\right\}$ is a 4-frame of characteristic $q$ as well as characteristic $p$. In this frame $\alpha_{15}$ (cf. (1.5)) is given by

$$
\alpha_{15}(x)=\left(\left(x+b_{2}\right)\left(d_{12}+b_{5}\right)+d_{52}\right)\left(b_{1}+b_{5}\right) .
$$

Thus $\alpha_{15}^{p}\left(b_{1}\right)=b_{1}$ and $\alpha_{15}^{q}\left(b_{1}\right)=b_{1}$. Since $p$ and $q$ are relatively prime, it follows that $\alpha_{15}\left(b_{1}\right)=b_{1}$. However, by Lemma 1.4, $\alpha_{15}\left(b_{1}\right)=d_{15}$. Thus $b_{1}=$ $d_{15}$, a contradiction.

The above proof yields the following corollary.

COROllaRy 3.2. Let $M$ be a modular lattice containing 4-frames $\left\{a_{i}, c_{1 j}\right\}$ and $\left\{a_{i}^{\prime}, c_{1 j}^{\prime}\right\}$ of characteristics $p$ and $q$, respectively, for distinct primes $p$ and $q$. Also suppose that $a_{1}+a_{2} / a_{1} a_{2} \nearrow a_{1}^{\prime}+a_{2}^{\prime} / a_{1}^{\prime} a_{2}^{\prime}$ and $a_{1}^{\prime}=a_{1}+a_{1}^{\prime} a_{2}^{\prime}, a_{2}^{\prime}=a_{2}+$ $a_{1}^{\prime} a_{2}^{\prime}$, and $c_{12}^{\prime}=c_{12}+a_{1}^{\prime} a_{2}^{\prime}$. Then $a_{1} / a_{1} a_{2}$ is a distributive sublattice of $M$.

We shall use this corollary and the lattice $L$ of $\$ 2$ to show that epimorphisms in the category of modular lattices need not be onto. Let $A=\{(x, y) \in L \times L: x \leqslant y\}$ and let $B$ be the sublattice of $L \times L$ generated by $A$ and $\left(a_{1}, 0\right)$. Let $f$ be the natural embedding of $A$ into $B$. We shall show that $f$ is an epimorphism, although it clearly is not onto. Let $g$ be a homomorphism from $B$ to a modular lattice $C$. We shall show that $g$ is determined by its values on $A$. It then follows easily from the categorical definition of epimorphism that $f$ is an epimorphism. Since $B$ is generated by $A$ and $\left(a_{1}, 0\right), g$ is determined by its values on $A$ and $\left(a_{1}, 0\right)$. The lattice $L$ is embedded into $A$, and hence into $B$ by the diagonal embedding, $x \rightarrow(x, x)$. Thus $B$ contains elements $\left(\left(a_{i}, a_{i}\right)\right.$, etc. $)$ satisfying the hypotheses of Corollary 3.2. Since these hypotheses are preserved by homomorphisms, this corollary implies that $g\left(a_{1}, a_{1}\right) / g(0,0)$ in $C$ is distributive. Now $\left(a_{1}, 0\right)$ is a relative complement of $\left(0, a_{1}\right)$ in the quotient $\left(a_{1}, a_{1}\right) /(0,0)$. Thus $g\left(a_{1}, 0\right)$ is determined by $g\left(0, a_{1}\right)$ since relative complements are unique in distributive lattices. Hence $g$ is determined by its restriction to $A$. Thus we have proved the following theorem.

THEOREM 3.3. Epimorphisms in the category of modular lattice and lattice homomorphisms are not necessarily onto.

THEOREM 3.4. FM(5) is not residually finite, and there is a lattice identity with five variables which holds in all finite modular lattices but not in all modular lattices.

Proof. First recall that $L$ depends on the choice of the fields $F$ and $K$ and the isomorphism $\sigma$ from $a_{1}+a_{2}+a_{3}+a_{4} / a_{3}+a_{4}$ to $a_{1}^{\prime}+a_{2}^{\prime} / 0^{\prime}$. The proof of Theorem 2.1 depends only on $L$ having the two frames $\left\{a_{i}, c_{1 j}\right\}$ and $\left\{a_{i}^{\prime}, c_{1 j}^{\prime}\right\}$. Thus we need really only consider the sublattice $L^{\prime}$ of $L$ generated 
by $\left\{a_{i}, c_{1 j}, a_{i}^{\prime}, c_{1 j}^{\prime}: i=1,2,3,4, j=2,3,4\right\}$. It is easy to see that $L^{\prime}$ has the same form as $L$, for certain subfields $F^{\prime} \subseteq F$ and $K^{\prime} \subseteq K$. Thus we may assume that $L$ is generated by $\left\{a_{i}, c_{1 j}, a_{i}^{\prime}, c_{1 j}^{\prime}\right\}$. We mention that for any fields $F$ and $K$ such that $|F|=|K|=\omega$ and $\operatorname{char}(F)=p \neq q=\operatorname{char}(K)$ one can choose the map $\sigma$ so that the $L$ thus obtained is generated by $\left\{a_{i}, c_{1 j}, a_{i}^{\prime}, c_{1 j}^{\prime}\right\}$.

We shall complete the proof by showing that $a_{i}, c_{1 j}, a_{i}^{\prime}$, and $c_{1 j}^{\prime}$ are in the sublattice of $L$ generated by

$$
G=\left\{a_{3}^{\prime}+a_{4}^{\prime}, c_{23}^{\prime}, c_{13}^{\prime}+c_{24}^{\prime}, a_{1}+a_{2}, c_{13}+c_{24}\right\}
$$

First

$$
\left(a_{3}^{\prime}+a_{4}^{\prime}\right) c_{23}^{\prime}=\left(a_{3}^{\prime}+a_{4}^{\prime}\right)\left(a_{2}^{\prime}+a_{3}^{\prime}\right) c_{23}^{\prime}=a_{3}^{\prime} c_{23}^{\prime}=0^{\prime} \text {. }
$$

Thus $0^{\prime} \in\langle G\rangle$. Hence $a_{1}+a_{2}+0^{\prime}=a_{1}^{\prime}+a_{2}^{\prime} \in\langle G\rangle$. Now

$$
\left(c_{23}^{\prime}+a_{3}^{\prime}+a_{4}^{\prime}\right)\left(a_{1}^{\prime}+a_{2}^{\prime}\right)=\left(a_{2}^{\prime}+a_{3}^{\prime}+a_{4}^{\prime}\right)\left(a_{1}^{\prime}+a_{2}^{\prime}\right)=a_{2}^{\prime} \in\langle G\rangle
$$

and

$$
\left(c_{23}^{\prime}+a_{1}^{\prime}+a_{2}^{\prime}\right)\left(a_{3}^{\prime}+a_{4}^{\prime}\right)=a_{3}^{\prime} \in\langle G\rangle .
$$

Moreover,

$$
\begin{aligned}
\left(c_{13}^{\prime}+c_{24}^{\prime}\right)\left(c_{23}^{\prime}+a_{1}^{\prime}+a_{2}^{\prime}\right) & =\left(c_{13}^{\prime}+c_{24}^{\prime}\right)\left(a_{1}^{\prime}+a_{2}^{\prime}+a_{3}^{\prime}\right) \\
& =c_{13}^{\prime}+c_{24}^{\prime}\left(a_{1}^{\prime}+a_{2}^{\prime}+a_{3}^{\prime}\right)=c_{13}^{\prime} \in\langle G\rangle,
\end{aligned}
$$

and

$$
c_{24}^{\prime}=\left(c_{13}^{\prime}+c_{24}^{\prime}\right)\left(c_{23}^{\prime}+a_{3}^{\prime}+a_{4}^{\prime}\right) \in\langle G\rangle .
$$

Now

$$
\begin{gathered}
a_{1}^{\prime}=\left(a_{1}^{\prime}+a_{2}^{\prime}\right)\left(c_{13}^{\prime}+a_{3}^{\prime}\right), \quad a_{4}^{\prime}=\left(a_{3}^{\prime}+a_{4}^{\prime}\right)\left(c_{24}^{\prime}+a_{2}^{\prime}\right), \\
c_{12}^{\prime}=\left(c_{13}^{\prime}+c_{23}^{\prime}\right)\left(a_{1}^{\prime}+a_{2}^{\prime}\right) \quad \text { and } \quad c_{14}^{\prime}=\left(c_{12}^{\prime}+c_{24}^{\prime}\right)\left(a_{1}^{\prime}+a_{2}^{\prime}\right)
\end{gathered}
$$

are all in $\langle G\rangle$. Thus $\left\{a_{i}^{\prime}, c_{1 j}^{\prime}\right\} \subseteq\langle G\rangle$. Now

$$
a_{1}+a_{2}+c_{13}+c_{24}=a_{1}+a_{2}+a_{3}+a_{4}=1 \in\langle G\rangle
$$

so $a_{3}+a_{4}=1 \cdot 0^{\prime} \in\langle G\rangle$. Also $a_{1}=a_{1}^{\prime}\left(a_{1}+a_{2}\right), a_{2}=a_{1}^{\prime}\left(a_{1}+a_{2}\right)$, and $c_{12}$ $=c_{12}^{\prime}\left(a_{1}+a_{2}\right)$ are in $\langle G\rangle$. So

$$
\left(c_{13}+c_{24}+a_{1}\right)\left(a_{3}+a_{4}\right)=\left(a_{1}+a_{3}+c_{24}\right)\left(a_{3}+a_{4}\right)=a_{3} \in\langle G\rangle .
$$

Similarly

$$
\begin{gathered}
a_{4}=\left(c_{13}+c_{24}+a_{2}\right)\left(a_{3}+a_{4}\right), \quad c_{34}=\left(c_{12}+c_{13}+c_{24}\right)\left(a_{3}+a_{4}\right), \\
c_{13}=\left(c_{13}+c_{24}\right)\left(a_{3}+a_{4}+a_{1}\right),
\end{gathered}
$$

and

$$
c_{24}=\left(c_{13}+c_{24}\right)\left(a_{3}+a_{4}+a_{2}\right)
$$

are in $\langle G\rangle$. Finally $c_{14}=\left(a_{1}+a_{4}\right)\left(c_{13}+c_{34}\right)$ is in $\langle G\rangle$. Thus $a_{1}, a_{2}, a_{3}$, $a_{4}, c_{12}, c_{13}$, and $c_{14}$ are all in $\langle G\rangle$, showing that $L$ is five-generated. The theorem now follows. 
It is possible to construct explicitly the identity alluded to in the above theorem. Let $f$ be a homomorphism from $F M(5)$ onto $L$. As in the proof of Theorem 2.1, it is possible to choose $\bar{a}_{i}, \bar{c}_{1 j}, \bar{a}_{i}^{\prime}, \bar{c}_{1 j}^{\prime} \in F M(5)$ such that $f\left(\bar{a}_{i}\right)=$ $a_{i}$, and $\left\{\bar{a}_{i}, \bar{c}_{1 j}\right\},\left\{\bar{a}_{i}^{\prime}, \bar{c}_{1 j}^{\prime}\right\}$ are 4-frames of characteristic $p$ and $q$, and the sublattice generated by $\left\{\bar{a}_{i}, \bar{a}_{i}^{\prime}\right\}$ is $D$. The proof began by choosing inverse images of $a_{i}$, etc., and modifying these, eventually obtaining the $\bar{a}_{i}$, etc. Each of these modifications can be explicitly carried out. In this manner a term for $\bar{a}_{i}$ can be constructed. By the last part of the proof of Theorem 2.1, the equation $\bar{a}_{1}=\bar{a}_{2}$ holds in all finite modular lattices but fails in $L$.

We close this paper with some open problems. The first one was suggested to the author by Kirby Baker. Is the variety of modular lattices generated by its finite dimensional members? (Cf. [6].) Does $F M(\omega)$ have any infinite, subdirectly irreducible sublattices? e.g. $M_{\omega}$ ? The existence of such a sublattice would of course imply that $\Re$ is not generated by its finite members and if such a sublattice had infinite length the answer to the first problem would be no. Are there any infinite subdirectly irreducible projective modular lattices? It is possible to show that the rational projective plane is projective in the variety it generates, although it is not projective in $\Re$. Are the finitely generated free modular lattices weakly atomic? (Weakly atomic means that every proper quotient contains a prime quotient. Alan Day has proved the corresponding result for free lattices.) Is every splitting modular lattice (cf. [16]) finite? The results of this paper show that the answer to at least one of the last two questions must be no.

\section{REFERENCES}

1. K. Baker, Equational classes of modular lattices, Pacific J. Math. 28 (1969), 9-15.

2. P. Crawley and R. P. Dilworth, Algebraic theory of lattices, Prentice-Hall, Englewood Cliffs, N. J., 1973.

3. R. A. Dean, Component subsets of the free lattice on $n$ generators, Proc. Amer. Math. Soc. 7 (1956), 220-226.

4. R. P. Dilworth, The arithmetical theory of Birkhoff lattices, Duke Math. J. 8 (1941), 286-299.

5. R. Freese, Planar sublattices of FM(4), Algebra Universalis 6 (1976), 69-72.

6. Some varieties of modular lattices not generated by their finite dimensional members, Contributions to Universal Algebra, Colloq. Math. Soc. János Bolyai, vol. 17, North-Holland, Amsterdam, 1977, pp. 133-144.

7. F. Galvin and B. Jónsson, Distributive sublattices of a free lattice, Canad. J. Math. 13 (1961), 265-272.

8. G. Grätzer, B. Jónsson and H. Lakser, The amalgamation property in equational classes of lattices, Pacific J. Math. 45 (1973), 507-524.

9. M. Hall and R. P. Dilworth, The embedding problem for modular lattices, Ann. of Math (2) 45 (1944), 450-456.

10. C. Herrmann and A. Huhn, Lattices of normal subgroups which are generated by frames, Lattice Theory, Colloq. Math. Soc. János Bolyai, vol. 14, North-Holland, Amsterdam, 1976, pp. 97-136.

11. A. P. Huhn, Schwach distributive Verbände. I, Acta Sci. Math. (Szeged) 33 (1972), 297-305.

12. , On G. Gratzer's problem concerning automorphisms of a finitely presented lattice, Algebra Universalis 5 (1975), 65-71. 
13. B. Jónsson, Algebras whose congruence lattice is distributive, Math. Scand. 21 (1967), 110-121.

14. ___ Varieties of lattices: some open problems, (to appear).

15. F. Maeda, Kontimuierlishe Geometrien, Springer-Verlag, Berlin, 1958.

16. R. McKenzie, Equational bases and non-modular lattice varieties, Trans. Amer. Math. Soc. 174 (1972), 1-43.

17. A. Mitschke and R. Wille, Finite distributive lattices projective in the class of all modular lattices, Algebra Universalis 6 (1976), 383-393.

18. E. T. Schmidt, On finitely generated simple modular lattices, Period. Math. Hungar. 6 (1975), 213-216.

19. J. von Neumann, Continuous geometry, (I. Halperin, ed.), Princeton Univ. Press, Princeton, N. J., 1960.

20. R. Wille, Primitive Länge und primitive Weite bei modularen Verbände, Math. Z. 108 (1969), 129-136.

Department of Mathematics, University of HawaI, Honolulu, Hawan 96822 